\title{
Analysis of traditional knowledge of medicinal plants from residents in Gayasan National Park (Korea)
}

\author{
Mi-Jang Song ${ }^{1}$, Hyun Kim² ${ }^{2 *}$ Byoung-Yoon Lee ${ }^{3}$, Heldenbrand Brian ${ }^{4}$, Chan-Ho Park ${ }^{3}$ and Chang-Woo Hyun ${ }^{3}$
}

\begin{abstract}
Background: The purpose of this study is to investigate and analyze the traditional knowledge of medicinal plants used by residents in Gayasan National Park in order to obtain basic data regarding the sustainable conservation of its natural plant ecosystem.

Methods: Data was collected using participatory observations and in-depth interviews, as the informants also become investigators themselves through attending informal meetings, open and group discussions, and overt observations with semi-structured questionnaires. Quantitative analyses were accomplished through the informant consensus factor (ICF), fidelity level, and inter-network analysis (INA).

Results: In total, 200 species of vascular plants belonging to 168 genera and 87 families were utilized traditionally in 1,682 ethnomedicianal practices. The representative families were Rosaceae (6.5\%) followed by Asteraceae (5.5\%), Poaceae (4.5\%), and Fabaceae (4.0\%). On the whole, 27 kinds of plant-parts were used and prepared in 51 various ways by the residents for medicinal purposes. The ICF values in the ailment categories were muscular-skeletal disorders (0.98), pains (0.97), respiratory system disorders (0.97), liver complaints (0.97), and cuts and wounds (0.96). In terms of fidelity levels, 57 plant species showed fidelities levels of 100\%. Regarding the inter-network analysis (INA) between ailments and medicinal plants within all communities of this study, the position of ailments is distributed into four main groups.

Conclusion: The results of the inter-network analysis will provide a suitable plan for sustainable preservation of the national park through a continued study of the data. Particular species of medicinal plants need to be protected for a balanced plant ecosystem within the park. Consequently, through further studies using these results, proper steps need to be established for preparing a wise alternative to create a sustainable natural plant ecosystem for Gayasan National Park and other national parks.
\end{abstract}

Keywords: Traditional knowledge, Informant consensus factor, Fidelity level, Inter-network analysis, Gayasan National Park

\section{Introduction}

National Parks in the world grow various useful bioresources owing to its well-preserved natural plant ecosystem. For residents living within a national park, these resources become materials for medicine, food, clothing, and other purposes. The relationship between

\footnotetext{
* Correspondence: hyunk@jj.ac.kr

${ }^{2}$ School of Alternative Medicine and Health Science, Jeonju University, 303 Cheonjam-ro, Wansan-gu, Jeonju 560-759, Republic of Korea

Full list of author information is available at the end of the article
}

the natural conservation of a national park and the life of its residents is interconnected [1].

Also, the traditional knowledge regarding the bioresources of residents living in a national park affects the natural conservation of an ecosystem [2]. Among all knowledge, the traditional knowledge of food and medicinal plants causes negative effects to plant ecosystems because of its applicability. Particularly, the additional value of medicinal plants is a major concern for species possessing high efficacy and utility as they are potentially overused and supplies become rapidly depleted [3]. These 
trends are stronger in developing countries with poor health care systems than in developed countries [4].

From this point, the analysis and investigation of traditional knowledge for medicinal plants used by residents in a national park will be applied for a sustainable conservation plan for a natural plant ecosystem.

Research in this field has been widely accomplished in several countries around the world, including Europe [5,6], Africa [7,8], Asia [9,10], North America [11], and South America [12]. The results of this research have been utilized as basic data to formulate a sustainable conservation plan for natural plant ecosystems within a national park.

In Korea, the research of the same pattern has been only conducted from residents living in Hallasan National Park [13].

The natural plant ecosystem of Gayasan National Park, located in the southeast region of Korea has been well preserved. Residents have maintained a traditional culture for over 30 years. These residents have utilized various ethnomedicine for treating numerous ailments. Therefore, a balance between medicinal plants and their utilization plays a very important role to sustain the natural conservation of a national park.

This study aims to investigate and analyze the traditional knowledge of medicinal plants used by residents in Gayasan National Park in order to obtain basic data regarding the sustainable conservation of its natural plant ecosystem.

\section{Research area and methods}

\section{Climate and geography of Gayasan National Park}

The study area is located in the center of the southern region of Korea and lies between $35^{\circ} 45^{\prime} \mathrm{N}$ to $35^{\circ} 51^{\prime} \mathrm{N}$ latitude and $128^{\circ} 02^{\prime} 30^{\prime \prime} \mathrm{E}$ to $128^{\circ} 09^{\prime} 30^{\prime \prime} \mathrm{E}$ longitude (Figure 1). The study area measures $77.256 \mathrm{~km}^{2}$ in areas including two provinces, one city, and four counties in its administrative district [14]. The annual average temperature is $13^{\circ} \mathrm{C}$ and the annual average precipitation is approximately $1,275.6 \mathrm{~mm}$ [15].

\section{Investigative method}

Proper data was collected using participatory observations and in-depth interviews, as the informants also become investigators themselves through attending informal meetings, open and group discussions, and overt observations with semi-structured questionnaires [16,17].

The content of the semi-structured questionnaires was composed of diverse information about medicinal plants, including local names, plant-parts used, ailments, methods of preparation, manufacturing and administration, dosage, and the usable duration regarding each curable formula [17-19].

All plant specimens were collected during their flowering or fruiting seasons and were organized utilizing the normal specimen manufacturing method $[18,20]$. The voucher specimens were deposited for preservation in the herbarium of Jeonju University. The precise identification of plants mentioned by the informants was performed in accordance with Lee [21] and Lee [22]. Scientific names of plants were confirmed by the National Knowledge and Information System for Biological Species [23] of Korea.

\section{Quantitative analysis}

\section{Informant consensus factor (ICF)}

The ICF was used to analyze the agreement degree of the informants' knowledge about each category of ailments $[24,25]$. The ICF was calculated using the following formula:

$$
\mathrm{ICF}=(n u r-n t) /(n u r-1),
$$

where nur stands for the number of use reports of informants for a particular illness category, and $n t$ is the number of species used by all informants for a particular illness.

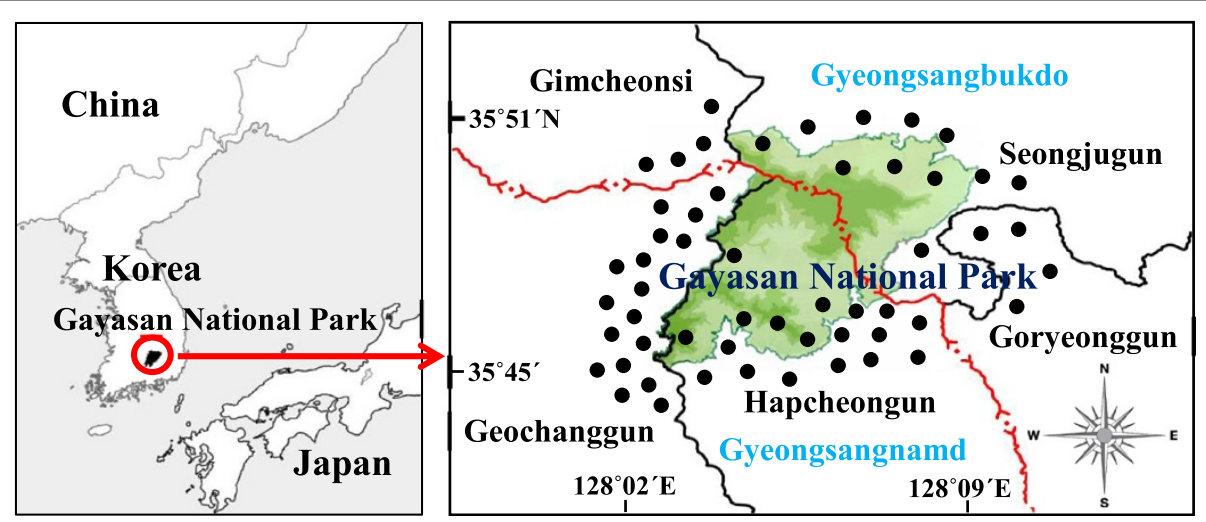

Figure 1 Investigation sites. 


\section{Fidelity level (FL)}

The FL was employed to determine the most important plant species used for treating certain diseases by the local herbal practitioners and elderly people living in the study area $[17,20,26]$. The FL was calculated using the following formula:

$$
\mathrm{FL}(\%)=\mathrm{Np} \times 100 / \mathrm{N}
$$

where $\mathrm{Np}$ is the number of informants that mentioned the specific plant species used to treat certain ailments, and $\mathrm{N}$ is the total number of the informants who utilized the plants as medicine for treating any given ailment.

\section{Inter-network analysis (INA)}

The INA considers the results of the interrelationship among each individual of a community instead of focusing on the independent characteristics of an individual within the community. The INA has been applied within communities for various ethnographical problems, including ethnogenesis [27] and obesity [28-30].

However, the INA has yet to be applied to traditional medicinal knowledge, although it has been included in relation to its ethnographical properties.

Therefore, this research has newly applied this method in order to attain more network information between categories of ailments and medicinal plants within communities in Korea. The results of the INA were analyzed using UCINET (Ver. 6.460) and NetDraw (Ver. 2.125) software [31,32].

\section{Results and discussion}

\section{Demographic characteristics of the informants}

Field investigations were conducted from May 2012 to October 2012. All 208 key informants (28 men and 180 women) were randomly selected at 54 sites, which included community halls, the senior welfare centers, and the traditional markets. The average age of the informants was 77 years of age, with a range in age from 52 to 93 , with residents living more than 30 years in the study area (Table 1).

\section{Ethnomedicinal knowledge}

In total, 200 species of vascular plants belonging to 168 genera and 87 families were utilized traditionally in 1,682 ethnomedicianal practices.

The species numbers from the Gayasan National Park were similar to those of other communities from previous research, including the communities of the western region of Jeolla North Province (183 species), the southern mountainous region (217 species) of Korea, and Hallasan National Park $[13,16,19]$. These results conclude that people living in the communities in and around Gayasan National Park possess similar application abilities for
Table 1 Demographic characteristics

\begin{tabular}{lc}
\hline Gender & \\
\hline Male & $28(13.5 \%)$ \\
\hline Female & $180(86.5 \%)$ \\
\hline Age & $1(0.5 \%)$ \\
\hline $50-59$ & $21(10.1 \%)$ \\
\hline $60-69$ & $106(51.0 \%)$ \\
\hline $70-79$ & $74(35.6 \%)$ \\
\hline $80-89$ & $6(2.9 \%)$ \\
\hline $90-99$ & \\
\hline Educational attainment & $154(74.0 \%)$ \\
\hline Never attended school & $13(6.3 \%)$ \\
\hline Attended school for less than 6 years & $17(8.2 \%)$ \\
\hline Attended school for 6 years & $14(6.7 \%)$ \\
\hline Finished middle school & $10(4.8 \%)$ \\
\hline Finished high school &
\end{tabular}

ethnomedicinal knowledge with other communities in Korea. Also, the number of species recorded in Gayasan National Park occupied 36.9\% of the flora (542 species) in the study area [15]. This high percentage means that all medicinal plants collected by residents may negatively affect the conservation of a natural plant ecosystem.

Regarding the number of species in their families and the percentage of the number of times mentioned from the our current investigations, 13 species of Rosaceae occupied $6.5 \%$ of the total species mentioned, followed by 12 species of Asteraceae, 11 species of Poaceae, and 9 species of Fabaceae, which occupied 5.5\%, 4.5\% and 4.0\% of the whole, respectively. Generally, these four families contain many more medicinal species than any other family. This data is similar to results obtained within national parks of other countries, including Pollino National Park in Italy [5], Kibale National Park in Uganda [8], Ben En National Park in Vietnam [9], Cumbres de Monterrey National Park in Mexico [11], and IsiboroSecure National Park in Bolivia [12].

Our overall analysis reveals that 27 plant-parts were selected as medicinal materials. Stems were the most frequently used plant-parts, constituting $25.7 \%$ of the whole followed by roots $(22.7 \%)$, fruits $(12.9 \%)$, whole parts $(7.3 \%)$, seeds $(5.3 \%)$, and other sections of the plant. These results were similar to data obtained in Korea for the western region of Jeolla North Province [19], the southern mountainous regions [16], and Hallasan National Park [13].

The results revealed 51 modes of preparation for the medicinal materials. The percentages for the main modes of preparation were as follows: infusions (32.1\%), 
sweet drinks made from fermented rice (20.1\%), brewings (9.3\%), macerations (7.1\%), and juices (6.8\%). Oral administration accounted for $84.8 \%$ of all applications, while topical application totaled $15.2 \%$.

Among the medicinal plants mentioned more than 50 times, with certain species used to treat numerous ailments, Hordeum vulgare var. hexastichon (L.) Asch. was used to treat 36 ailments, followed by Artemisia princeps Pamp. in treating 24 ailments, and Zanthoxylum schinifolium Siebold. \& Zucc. for treating 11 ailments. One area of major concern is the over application of the Artemisia princeps Pamp. and Zanthoxylum schinifolium Siebold. \& Zucc. which can easily lead to its extinction within the plant ecosystem.

\section{Ethno-veterinary knowledge}

The medicinal plants for ethno-veterinary treatments were recorded as 15 families, 22 genera, and 23 species that displayed 38 ethnomedicinal practices (Table 2). Among the veterinary medicinal plants, the whole part of Artemisia princeps Pamp., Papaver somniferum L., Picrasma quassioides (D.Don) Benn., Sanguisorba officinalis L., and Poria cocos Wolf were used most often to treat bovine diarrhea. Particularly, Ipomoea batatas (L.) Lam., Ricinus communis L., and Zanthoxylum schinifolium Siebold. \& Zucc. were ingested for particular pig ailments, while Lageneria leucantha Rushy was generally used for treating a bovine disease caused by a loss of appetite.

At present, these veterinary species do not affect the natural plant ecosystem of a national park anymore, as residents use a modern system of veterinary medicine to treat the diseases of domestic animals.

\section{Quantitative analysis}

ICF

The ICF ranges from 0 to 1 , where increasing values of this factor indicate a higher rate of informant consensus among the illness category.

The category with the highest degree of consensus from the informants was muscular-skeletal disorders (0.98). The ranking followed with remarks from informants related to concerns of pain (0.97), respiratory system disorders (0.97), liver complaints (0.97), and cuts and wounds (0.96). The lowest degree of consensus was birth-related disorders (Table 3 ). These results expose the fact that the residents living in a national park work in dry-field farming and laboriously gather products from the forests as they live in the mountainous region.

Medicinal plants for treating disease with a higher degree of consensus can easily be harvested for treating medical ailments. As a result, the natural plant ecosystem of a national park may be partly destroyed in the near future. Therefore, through further study, proper steps to determine a wise alternative to protect them need to be considered.

\section{FL}

The FL is useful for identifying the key informants' most preferred species in use for treating certain ailments. The FL values in this study varied from $1.0 \%$ to $100 \%$. Generally, the FL of $100 \%$ for a specific plant indicates that all of the use-reports mentioned the same plant for specific treatment [33].

This study classified 57 species of plants with a FL of $100 \%$, even without considering plants that were mentioned only once for better accuracy (Table 3). This information reveals that the informants had a tendency to rely on one specific plant species for treating one specific ailment than for several different ailments. These species possess a much higher potential for being gathered in Gayasan National Park.

Special attention for species conservation within the national park was given to important species $(\mathrm{N}, \mathrm{Np})$ with a FL above $100 \%$, regarding the viewpoint of the number of times mentioned and the consensus level for the specific ailment, like Solanum nigram L. $(68,68)$ in being used for burns, Fallopia japonica (Houtt.) RonseDecr. for pollakiuria $(60,60)$, Colocassia esculenta (L.) Schott $(15,15)$ for sterility, Euonymus sachalinensis (F. Schmidt) Maxim. $(13,13)$ for hemorrhoids, and Staphylea bumalda DC. $(13,13)$ for facial nerve paralysis (Table 2).

INA between categories of ailments and medicinal plants INA has originally analyzed social phenomenon and its trends through the network of components [34]. Our research has attempted to analyze the interrelationship between aliments and medicinal plants recorded within these communities.

In relation to the network of ailments and medicinal plants, the position of the ailments is distributed into four main groups, where each circle represents four different groups of ailments (Figure 2).

Accordingly, the first group is positioned in the upper section of Figure 2 and consists of poisonings, respiratory system disorders, others, inflammation and so on.

The second group is located in the bottom left section and consists of nervous system disorders, gastrointestinal disorders, cuts and wounds, and pains. This group of disorders will require a deeper analysis in order to determine the overlapping use of medicinal species for these ailments.

The third group is positioned on the left side and consists of birth-related disorders and genitourinary system disorders. These results depict that the same medicinal 
Table 2 Plant species for medicinal purposes in community of Gayasan National Park in Korea

\begin{tabular}{|c|c|c|c|c|c|c|c|c|c|}
\hline Family & Scientific name & Voucher & Abbreviation $^{*}$ & Korean name & Used part & Ailments & Preparation & Application & $\mathrm{FL}$ \\
\hline Aceraceae & $\begin{array}{l}\text { Acer pictum subsp. mono } \\
\text { (Maxim.) Ohashi }\end{array}$ & KH5340 & S1 & Gorosoenamu & Sap & Hypofunction & Sap & Oral & 100.00 \\
\hline Actinidiaceae & $\begin{array}{l}\text { Actinidia arguta (Siebold } \\
\& \text { Zucc.) Planch. ex Miq. }\end{array}$ & KH5341 & S5 & Darae & Stem & $\begin{array}{l}\text { Abdominal } \\
\text { pain }\end{array}$ & Infusion & Oral & 100.00 \\
\hline \multirow[t]{11}{*}{ Amaranthaceae } & $\begin{array}{l}\text { Achyranthes japonica } \\
\text { (Miq.) Nakai }\end{array}$ & KH5342 & S2 & Soemureup & Root & Arm pain & $\begin{array}{l}\text { A sweet drink made from fermented rice, } \\
\text { brewing, infusion }\end{array}$ & Oral & 16.71 \\
\hline & & & & & & Arthritis & Infusion & Oral & 1.62 \\
\hline & & & & & & Bone diseases & $\begin{array}{l}\text { A sweet drink made from fermented rice, } \\
\text { brewing, infusion }\end{array}$ & Oral & 32.61 \\
\hline & & & & & & Common cold & $\begin{array}{l}\text { A sweet drink made from fermented rice, } \\
\text { infusion }\end{array}$ & Oral & 2.43 \\
\hline & & & & & & Convulsion & $\begin{array}{l}\text { A sweet drink made from fermented rice, } \\
\text { brewing, infusion }\end{array}$ & Oral & 3.23 \\
\hline & & & & & & Knee pain & A sweet drink made from fermented rice & Oral & 2.16 \\
\hline & & & & & & Leg pain & $\begin{array}{l}\text { A sweet drink made from fermented rice, } \\
\text { brewing, decoction, infusion }\end{array}$ & Oral & 31.54 \\
\hline & & & & & & Lumbago & $\begin{array}{l}\text { A sweet drink made from fermented rice, } \\
\text { infusion }\end{array}$ & Oral & 9.16 \\
\hline & & & & & & Pollakiuria & Infusion & Oral & 0.54 \\
\hline & Celosia cristata L. & KH5343 & S41 & Maendeurami & Whole part & $\begin{array}{l}\text { Carpal tunnel } \\
\text { syndrome }\end{array}$ & Infusion & Oral & 50.00 \\
\hline & & & & & & $\begin{array}{l}\text { Tarsal tunnel } \\
\text { syndrome }\end{array}$ & Infusion & Oral & 50.00 \\
\hline \multirow[t]{8}{*}{ Anacardiaceae } & Rhus javanica L. & KH5344 & S155 & Bulknamu & Worm cyst & $\begin{array}{l}\text { Coated } \\
\text { tongue }\end{array}$ & Infusion & Topical & 15.12 \\
\hline & & & & & & Stomatitis & $\begin{array}{l}\text { Dried, infusion, maceration, powder, raw, } \\
\text { steamed }\end{array}$ & Oral, topical & 84.88 \\
\hline & Rhus verniciflua Stokes & KH5345 & S156 & Otnamu & Bark & $\begin{array}{l}\text { Abdominal } \\
\text { cold } \\
\text { hypersentivity }\end{array}$ & Infusion, simmer & Oral & 58.97 \\
\hline & & & & & & $\begin{array}{l}\text { Gastroenteric } \\
\text { disorder }\end{array}$ & Simmer & Oral & 19.87 \\
\hline & & & & & Lacquer & $\begin{array}{l}\text { Abdominal } \\
\text { cold } \\
\text { hypersentivity }\end{array}$ & Mixed in egg, roast, sap & Oral & 58.97 \\
\hline & & & & & & $\begin{array}{l}\text { Facial nerve } \\
\text { paralysis }\end{array}$ & Raw & Topical & 0.64 \\
\hline & & & & & Stem & $\begin{array}{l}\text { Abdominal } \\
\text { cold } \\
\text { hypersentivity }\end{array}$ & Infusion, simmer, soup & Oral & 58.97 \\
\hline & & & & & & Diarrhea & Burn, powder & Oral & 1.28 \\
\hline
\end{tabular}


Table 2 Plant species for medicinal purposes in community of Gayasan National Park in Korea (Continued)

\begin{tabular}{|c|c|c|c|c|c|c|c|c|c|}
\hline & & & & & & $\begin{array}{l}\text { Gastroenteric } \\
\text { disorder }\end{array}$ & Infusion, simmer & Oral & 19.87 \\
\hline & & & & & & Hangover & Infusion, simmer & Oral & 10.90 \\
\hline & & & & & & Indigestion & Infusion & Oral & 3.85 \\
\hline & & & & & & $\begin{array}{l}\text { Lack of } \\
\text { energy }\end{array}$ & Infusion & Oral & 1.92 \\
\hline & & & & & & $\begin{array}{l}\text { Woman } \\
\text { diseases }\end{array}$ & Infusion & Oral & 2.56 \\
\hline & & & & & Young leaf & $\begin{array}{l}\text { Gastroenteric } \\
\text { disorder }\end{array}$ & Raw & Oral & 19.87 \\
\hline \multirow[t]{16}{*}{ Apiaceae } & $\begin{array}{l}\text { Angelica acutiloba } \\
\text { (Siebold \& Zucc.) Kitag. }\end{array}$ & KH5346 & S14 & Waedanggwi & Root & $\begin{array}{l}\text { Poor } \\
\text { circulation }\end{array}$ & Infusion & Oral & 53.33 \\
\hline & & & & & & $\begin{array}{l}\text { Lack of } \\
\text { energy }\end{array}$ & Boiling, decoction & Oral & 46.67 \\
\hline & $\begin{array}{l}\text { Angelica dahurica (Fisch. } \\
\text { ex Hoffm.) Benth. \& Hook. } \\
\text { f. ex Franch. \& Sav. }\end{array}$ & KH5347 & S15 & Guritdae & Root & $\begin{array}{l}\text { Lack of } \\
\text { energy }\end{array}$ & Decoction & Oral & 100.00 \\
\hline & Angelica gigas Nakai & KH5348 & $\mathrm{S} 16$ & Chamdanggwi & Root & $\begin{array}{l}\text { Lack of } \\
\text { energy }\end{array}$ & Decoction, infusion & Oral, topical & 100.00 \\
\hline & Bupleurum falcatum L. & KH5349 & $\mathrm{S} 32$ & Siho & Root & Alopecia & A sweet drink made from fermented rice & Oral & 100.00 \\
\hline & Cnidium officinale Makino & KH5350 & S48 & Cheongung & Root & Bone diseases & A sweet drink made from fermented rice & Oral & 23.53 \\
\hline & & & & & & $\begin{array}{l}\text { Lack of } \\
\text { energy }\end{array}$ & Boiling, decoction & Oral, topical & 52.94 \\
\hline & & & & & & $\begin{array}{l}\text { Woman } \\
\text { diseases }\end{array}$ & Decoction & Oral & 23.53 \\
\hline & $\begin{array}{l}\text { Ledebouriella seseloides } \\
\text { (Hoffm.) H.Wolff }\end{array}$ & KH5351 & 599 & Bangpung & Root & Hypofunction & Decoction & Oral & 100.00 \\
\hline & $\begin{array}{l}\text { Oenanthe javanica } \\
\text { (Blume) DC. }\end{array}$ & KH5352 & S111 & Minari & Stem & Cancer & Juice & Oral & 25.00 \\
\hline & & & & & & Liver diseases & Juice & Oral & 29.17 \\
\hline & & & & & & Pus & Maceration & Topical & 25.00 \\
\hline & & & & & & Snakebite & Maceration & Topical & 20.83 \\
\hline & & & & & Whole part & Liver diseases & Juice & Oral & 29.17 \\
\hline & $\begin{array}{l}\text { Ostericum praeteritum } \\
\text { Kitag. }\end{array}$ & KH5353 & S115 & Ganghwal & Root & Hyperthermia & Infusion & Oral & 60.00 \\
\hline & & & & & & $\begin{array}{l}\text { Lack of } \\
\text { energy }\end{array}$ & Decoction & Oral & 40.00 \\
\hline \multirow[t]{2}{*}{ Araceae } & $\begin{array}{l}\text { Arisaema amurense f. } \\
\text { serratum (Nakai) Kitag. }\end{array}$ & KH5354 & $\mathrm{S} 19$ & Cheonnamseong & Corm & $\begin{array}{l}\text { Chronic } \\
\text { myofascial } \\
\text { pain }\end{array}$ & $\begin{array}{l}\text { Chopped noodles, clear soup with flour } \\
\text { dumplings }\end{array}$ & Oral & 55.56 \\
\hline & & & & & & & Maceration & Topical & 3.70 \\
\hline
\end{tabular}


Table 2 Plant species for medicinal purposes in community of Gayasan National Park in Korea (Continued)

\begin{tabular}{|c|c|c|c|c|c|c|c|c|c|}
\hline & & & & & & $\begin{array}{l}\text { Extravasated } \\
\text { blood }\end{array}$ & & & \\
\hline & & & & & & Milk fever & Clear soup with flour dumplings & Oral & 12.96 \\
\hline & & & & & & Tonsillitis & Clear soup with flour dumplings & Oral & 14.81 \\
\hline & & & & & & Wound & Maceration & Topical & 12.96 \\
\hline & & & & & Stem & Tonsillitis & Infusion & Oral & 14.81 \\
\hline & $\begin{array}{l}\text { Colocasia esculenta (L.) } \\
\text { Schott }\end{array}$ & KH5355 & S52 & Toran & Flower & Sterility & Infusion & Oral & 100.00 \\
\hline \multirow[t]{19}{*}{ Araliaceae } & $\begin{array}{l}\text { Aralia cordata var. } \\
\text { continentalis (Kitag.) Y.C. } \\
\text { Chu }\end{array}$ & KH5356 & S17 & Dokhwal & Root & $\begin{array}{l}\text { Abdominal } \\
\text { pain }\end{array}$ & Infusion & Oral & 5.56 \\
\hline & & & & & & Anorexia & Infusion & Oral & 5.56 \\
\hline & & & & & & Arthritis & Infusion & Oral & 11.11 \\
\hline & & & & & & Bone diseases & Brewing & Oral & 14.81 \\
\hline & & & & & & $\begin{array}{l}\text { Chronic } \\
\text { myofascial } \\
\text { pain }\end{array}$ & Brewing & Oral & 18.52 \\
\hline & & & & & & $\begin{array}{l}\text { Gastroenteric } \\
\text { disorder }\end{array}$ & Dissolution, maceration & Oral & 11.11 \\
\hline & & & & & & Lumbago & Brewing, maceration & Oral & 33.33 \\
\hline & Aralia elata (Miq.) Seem. & KH5357 & $\mathrm{S} 18$ & Dureupnamu & Root & Edema & Infusion & Oral & 100.00 \\
\hline & $\begin{array}{l}\text { Eleutherococcus senticosus } \\
\text { (Rupr. \& Maxim.) Maxim. }\end{array}$ & KH5358 & S66 & Gasiogalpi & Stem & Bone diseases & Decoction & Oral & 100.00 \\
\hline & $\begin{array}{l}\text { Eleutherococcus } \\
\text { sessiliflorus (Rupr. \& } \\
\text { Maxim.) S.Y.Hu }\end{array}$ & KH5359 & S67 & Ogalpinamu & Fruit & Bone diseases & Extraction & Oral & 47.38 \\
\hline & & & & & & Arm pain & $\begin{array}{l}\text { A sweet drink made from fermented rice, } \\
\text { brewing, infusion }\end{array}$ & Oral & 11.53 \\
\hline & & & & & & Arthritis & A sweet drink made from fermented rice & Oral & 1.05 \\
\hline & & & & & & Bone diseases & $\begin{array}{l}\text { A sweet drink made from fermented rice, } \\
\text { brewing, infusion }\end{array}$ & Oral & 47.38 \\
\hline & & & & & & Glycosuria & Infusion & Oral & 1.05 \\
\hline & & & & & & Growing pain & $\begin{array}{l}\text { A sweet drink made from fermented rice, } \\
\text { brewing }\end{array}$ & Oral & 2.94 \\
\hline & & & & & & Hyperthermia & Brewing & Oral & 0.21 \\
\hline & & & & & & Leg pain & $\begin{array}{l}\text { A sweet drink made from fermented rice, } \\
\text { brewing, infusion }\end{array}$ & Oral & 22.01 \\
\hline & & & & & & Liver diseases & Infusion & Oral & 1.89 \\
\hline & & & & & & Lumbago & $\begin{array}{l}\text { A sweet drink made from fermented rice, } \\
\text { infusion }\end{array}$ & Oral & 9.64 \\
\hline
\end{tabular}


Table 2 Plant species for medicinal purposes in community of Gayasan National Park in Korea (Continued)

\begin{tabular}{|c|c|c|c|c|c|c|c|c|c|}
\hline & & & & & & Neuralgia & Infusion & Oral & 0.42 \\
\hline & & & & & & Paralysis & Infusion & Oral & 1.26 \\
\hline & & & & & & Shoulder pain & A sweet drink made from fermented rice & Oral & 0.42 \\
\hline & & & & & & $\begin{array}{l}\text { Sinews and } \\
\text { joint pain }\end{array}$ & A sweet drink made from fermented rice & Oral & 0.21 \\
\hline & $\begin{array}{l}\text { Kalopanax septemlobus } \\
\text { (Thunb.) Koidz. }\end{array}$ & KH5360 & & Eumnamu & & Arm pain & A sweet drink made from fermented rice & Oral & 9.76 \\
\hline & & & & & & Bone diseases & Brewing, infusion & Oral & 55.10 \\
\hline & & & & & & Leg pain & A sweet drink made from fermented rice & Oral & 17.57 \\
\hline & & & & & Stem & $\begin{array}{l}\text { Abdominal } \\
\text { cold } \\
\text { hypersentivity }\end{array}$ & Infusion, simmer & Oral & 0.65 \\
\hline & & & & & & Arm pain & $\begin{array}{l}\text { A sweet drink made from fermented rice, } \\
\text { brewing, Infusion, Simmer }\end{array}$ & Oral & 9.76 \\
\hline & & & & & & Bone diseases & $\begin{array}{l}\text { A sweet drink made from fermented rice, } \\
\text { brewing, infusion, simmer }\end{array}$ & Oral & 55.10 \\
\hline & & & & & & $\begin{array}{l}\text { Gastroenteric } \\
\text { disorder }\end{array}$ & $\begin{array}{l}\text { A sweet drink made from fermented rice, } \\
\text { brewing }\end{array}$ & Oral & 3.04 \\
\hline & & & & & & Hyperthermia & Brewing & Oral & 0.22 \\
\hline & & & & & & Indigestion & Infusion & Oral & 0.22 \\
\hline & & & & & & $\begin{array}{l}\text { Lack of } \\
\text { energy }\end{array}$ & Infusion & Oral & 0.87 \\
\hline & & & & & & Leg pain & $\begin{array}{l}\text { A sweet drink made from fermented rice, } \\
\text { brewing, infusion, simmer }\end{array}$ & Oral & 17.57 \\
\hline & & & & & & Liver diseases & Infusion & Oral & 0.87 \\
\hline & & & & & & Lumbago & $\begin{array}{l}\text { A sweet drink made from fermented rice, } \\
\text { infusion }\end{array}$ & Oral & 9.11 \\
\hline & & & & & & Neuralgia & Infusion & Oral & 0.43 \\
\hline & & & & & & Paralysis & Brewing, infusion & Oral & 2.17 \\
\hline \multirow[t]{2}{*}{ Aristolochiaceae } & Asarum sieboldii Miq. & KH5361 & $\mathrm{S} 24$ & Jokdoripul & Root & Dental pain & Chew, dried & Topical & 22.22 \\
\hline & & & & & & Pain & $\begin{array}{l}\text { A sweet drink made from fermented rice, } \\
\text { infusion }\end{array}$ & Oral & 77.78 \\
\hline \multirow[t]{5}{*}{ Aspleniaceae } & $\begin{array}{l}\text { Athyrium yokoscense } \\
\text { (Franch. \& Sav.) H.Christ }\end{array}$ & KH5362 & $\mathrm{S} 26$ & Baemgosari & Stem & Abrasion & Maceration, rubbing & Topical & 13.41 \\
\hline & & & & & & Common cold & Infusion & Oral & 6.10 \\
\hline & & & & & & Hemorrhaging & Maceration, rubbing & Topical & 62.20 \\
\hline & & & & & & Snakebite & Maceration, raw & Topical & 15.85 \\
\hline & & & & & & Wound & Maceration & Topical & 2.44 \\
\hline
\end{tabular}


Table 2 Plant species for medicinal purposes in community of Gayasan National Park in Korea (Continued)

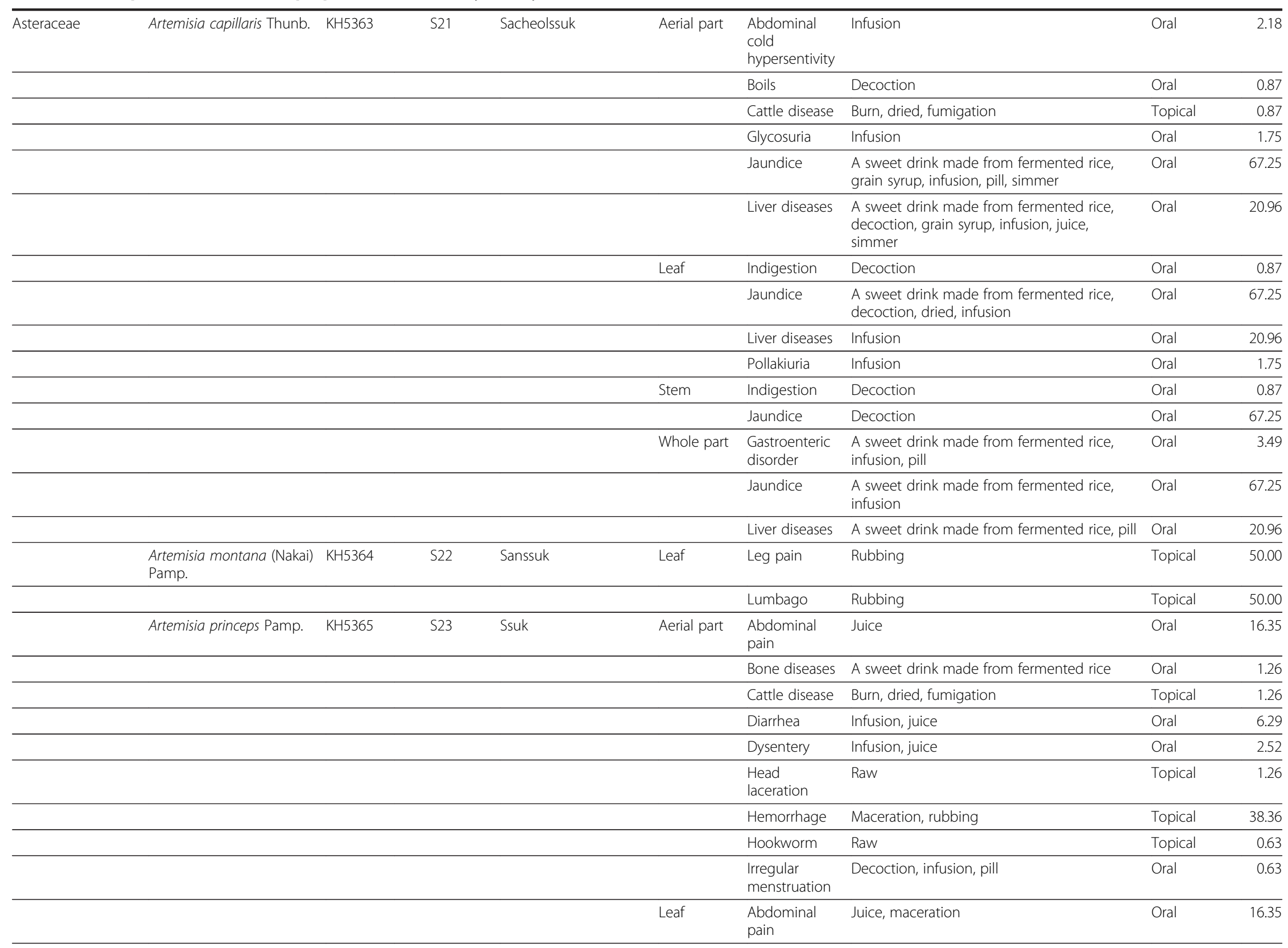


Table 2 Plant species for medicinal purposes in community of Gayasan National Park in Korea (Continued)

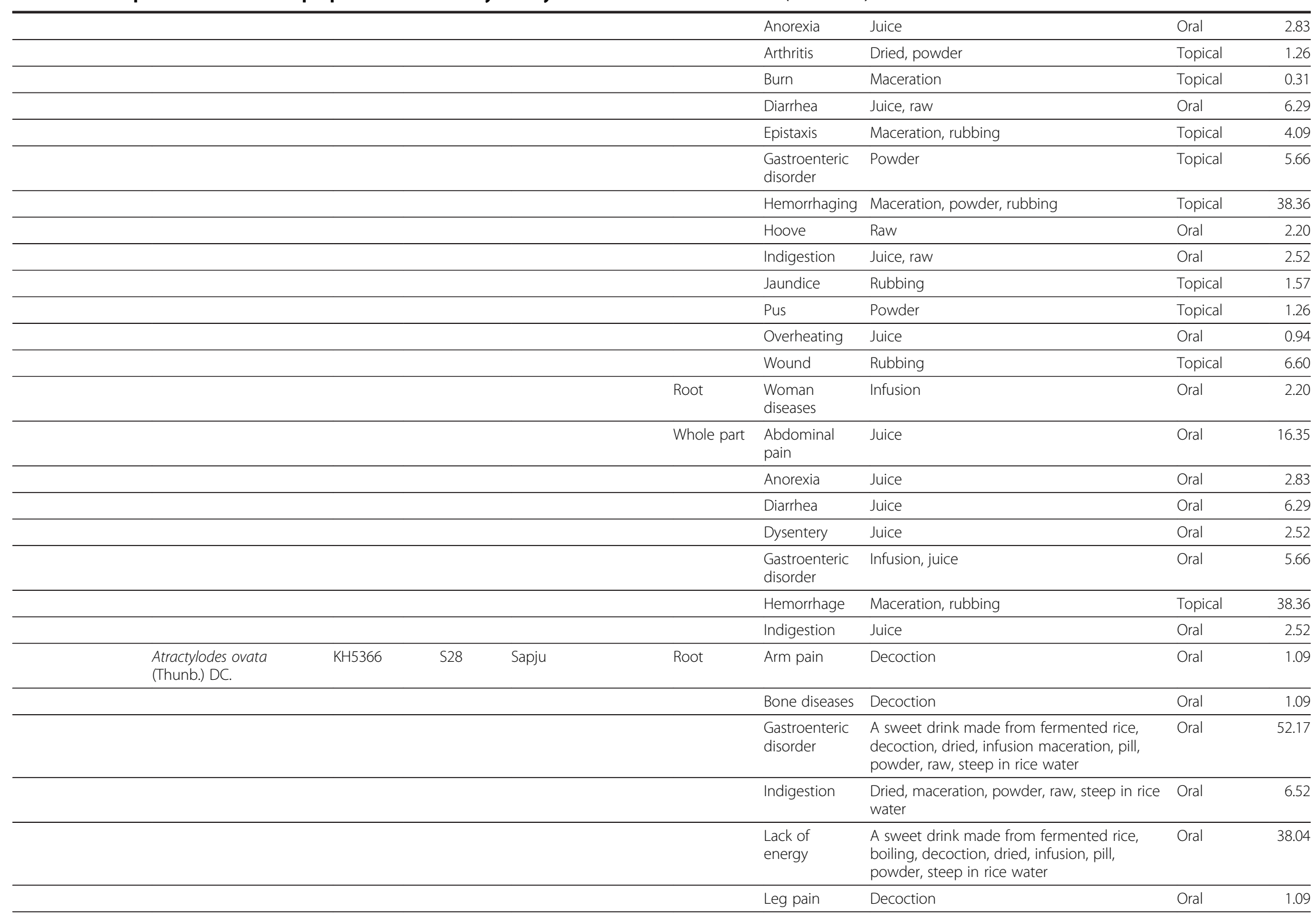


Table 2 Plant species for medicinal purposes in community of Gayasan National Park in Korea (Continued)

\begin{tabular}{|c|c|c|c|c|c|c|c|c|}
\hline Carthamus tinctorius $\mathrm{L}$. & KH5367 & $\mathrm{S} 37$ & Itggot & Seed & Bone diseases & A sweet drink made from fermented rice & Oral & 33.33 \\
\hline & & & & & Fracture & Boiling & Oral & 33.33 \\
\hline & & & & & Lumbago & A sweet drink made from fermented rice & Oral & 33.33 \\
\hline $\begin{array}{l}\text { Cirsium japonicum var. } \\
\text { maackii (Maxim.) Matsum. }\end{array}$ & KH5368 & S45 & Eonggeongkwi & Root & $\begin{array}{l}\text { Abdominal } \\
\text { cold } \\
\text { hypersentivity }\end{array}$ & Juice & Oral & 1.80 \\
\hline & & & & & $\begin{array}{l}\text { Abdominal } \\
\text { pain }\end{array}$ & Juice & Oral & 0.90 \\
\hline & & & & & Anhidrosis & Infusion, juice & Oral & 4.50 \\
\hline & & & & & Bone diseases & Juice & Oral & 0.90 \\
\hline & & & & & Epistaxis & Juice & Oral & 0.90 \\
\hline & & & & & $\begin{array}{l}\text { Gastroenteric } \\
\text { disorder }\end{array}$ & Juice & Oral & 0.90 \\
\hline & & & & & Hangover & Juice & Oral & 3.15 \\
\hline & & & & & Hookworm & Juice & Oral & 2.70 \\
\hline & & & & & $\begin{array}{l}\text { Lack of } \\
\text { energy }\end{array}$ & Juice & Oral & 3.60 \\
\hline & & & & & Lumbago & Infusion, juice & Oral & 4.50 \\
\hline & & & & & $\begin{array}{l}\text { Sexual } \\
\text { enhancement }\end{array}$ & Infusion, juice & Oral & 75.23 \\
\hline & & & & & Sterility & Infusion & Oral & 0.90 \\
\hline Helianthus tuberosus L. & KH5369 & $\mathrm{S} 86$ & Ddungddanji & Tuber & Glycosuria & Maceration, tea & Oral & 100.00 \\
\hline Lactuca sativa $\mathrm{L}$. & KH5370 & 597 & Sangchu & Seed & Oligogalactia & Infusion & Oral & 100.00 \\
\hline $\begin{array}{l}\text { Petasites japonicus } \\
\text { (Siebold \& Zucc.) Maxim. }\end{array}$ & KH5371 & S123 & Meowi & Leaf & $\begin{array}{l}\text { Gastroenteric } \\
\text { disorder }\end{array}$ & Infusion, wrapped in leaves & Oral & 27.59 \\
\hline & & & & & $\begin{array}{l}\text { Lacquer } \\
\text { poisoning }\end{array}$ & Maceration & Topical & 37.93 \\
\hline & & & & & Paralysis & Maceration & Oral & 10.34 \\
\hline & & & & Root & Convulsion & Maceration & Oral & 6.90 \\
\hline & & & & & Leg pain & Brewing & Oral & 17.24 \\
\hline & & & & Stem & $\begin{array}{l}\text { Gastroenteric } \\
\text { disorder }\end{array}$ & Seasoned cooked vegetables & Oral & 27.59 \\
\hline $\begin{array}{l}\text { Sigesbeckia glabrescens } \\
\text { (Makino) Makino }\end{array}$ & KH5372 & S175 & Jindeukchal & Aerial part & Pruritus & $\begin{array}{l}\text { A sweet drink made from fermented rice, } \\
\text { brewing, infusion, pill, simmer }\end{array}$ & Oral, topical & 79.89 \\
\hline & & & & Flower & Hypertension & Grain syrup, infusion & Oral & 3.45 \\
\hline & & & & & $\begin{array}{l}\text { Postpartum } \\
\text { myofascial } \\
\text { pain } \\
\text { syndrome }\end{array}$ & $\begin{array}{l}\text { A sweet drink made from fermented rice, } \\
\text { infusion }\end{array}$ & Oral & 11.49 \\
\hline
\end{tabular}


Table 2 Plant species for medicinal purposes in community of Gayasan National Park in Korea (Continued)

\begin{tabular}{|c|c|c|c|c|c|c|c|c|c|}
\hline & & & & & & Pruritus & Dried, mixed in liquor, maceration, pill & Oral & 79.89 \\
\hline & & & & & Fruit & Pruritus & Brewing, infusion & Oral, topical & 79.89 \\
\hline & & & & & Leaf & $\begin{array}{l}\text { Postpartum } \\
\text { myofascial } \\
\text { pain } \\
\text { syndrome }\end{array}$ & $\begin{array}{l}\text { A sweet drink made from fermented rice, } \\
\text { infusion }\end{array}$ & Oral & 11.49 \\
\hline & & & & & Whole part & Bone diseases & A sweet drink made from fermented rice & Oral & 5.17 \\
\hline & & & & & & Pruritus & $\begin{array}{l}\text { A sweet drink made from fermented rice, } \\
\text { infusion }\end{array}$ & Oral, topical & 79.89 \\
\hline & $\begin{array}{l}\text { Taraxacum platycarpum } \\
\text { Dahlst. }\end{array}$ & KH5373 & S184 & Mindeulre & Leaf & Liver diseases & $\begin{array}{l}\text { Dried, infusion, juice, Kimchi, raw, seasoned } \\
\text { cooked vegetables }\end{array}$ & Oral & 91.76 \\
\hline & & & & & Root & Cancer & Infusion & Oral & 8.24 \\
\hline & & & & & Whole part & Cancer & Dried, infusion & Oral & 8.24 \\
\hline & & & & & & Liver diseases & $\begin{array}{l}\text { A sweet drink made from fermented rice, } \\
\text { dried, extraction, infusion, juice }\end{array}$ & Oral & 91.76 \\
\hline & Xanthium strumarium L. & KH5374 & S195 & Doggomari & Aerial part & Boils & Infusion & Topical & 22.22 \\
\hline & & & & & Fruit & Pruritus & Infusion & Topical & 40.74 \\
\hline & & & & & Stem & Boils & Infusion & Topical & 22.22 \\
\hline & & & & & Whole part & Boils & Infusion & Topical & 22.22 \\
\hline & & & & & & $\begin{array}{l}\text { Gastroenteric } \\
\text { disorder }\end{array}$ & A sweet drink made from fermented rice & Oral & 11.11 \\
\hline & & & & & & Leg pain & A sweet drink made from fermented rice & Oral & 11.11 \\
\hline & & & & & & Pruritus & $\begin{array}{l}\text { A sweet drink made from fermented rice, } \\
\text { brewing, infusion }\end{array}$ & Oral, topical & 40.74 \\
\hline & & & & & & Skin diseases & $\begin{array}{l}\text { A sweet drink made from fermented rice, } \\
\text { brewing, infusion }\end{array}$ & Oral, topical & 14.81 \\
\hline \multirow[t]{2}{*}{ Balsaminaceae } & Impatiens balsamina $\mathrm{L}$. & KH5375 & 591 & Bongseonhwa & Whole part & Indigestion & Infusion & Oral & 59.26 \\
\hline & & & & & & Sterility & Decoction, infusion & Oral & 40.74 \\
\hline \multirow[t]{2}{*}{ Berberidaceae } & $\begin{array}{l}\text { Epimedium koreanum } \\
\text { Nakai }\end{array}$ & KH5376 & S68 & Samjigueopcho & Aerial part & $\begin{array}{l}\text { Sexual } \\
\text { enhancement }\end{array}$ & $\begin{array}{l}\text { A sweet drink made from fermented rice, } \\
\text { infusion }\end{array}$ & Oral & 57.14 \\
\hline & & & & & & Sterility & Tea & Oral & 42.86 \\
\hline \multirow[t]{4}{*}{ Betulaceae } & Betula costata Trautv. & KH5377 & S29 & Geojesunamu & Sap & Hypofunction & Sap & Oral & 35.71 \\
\hline & & & & & & Indigestion & Sap & Oral & 7.14 \\
\hline & & & & & Stem & Arm pain & A sweet drink made from fermented rice & Oral & 28.57 \\
\hline & & & & & & Leg pain & A sweet drink made from fermented rice & Oral & 28.57 \\
\hline \multirow[t]{4}{*}{ Bignoniaceae } & Catalpa ovata G.Don & KH5378 & S39 & Gaeodong & Stem & Bone diseases & A sweet drink made from fermented rice & Oral & 30.77 \\
\hline & & & & & & Indigestion & Infusion & Oral & 7.69 \\
\hline & & & & & & Leg pain & A sweet drink made from fermented rice & Oral & 30.77 \\
\hline & & & & & & Lumbago & A sweet drink made from fermented rice & Oral & 30.77 \\
\hline
\end{tabular}


Table 2 Plant species for medicinal purposes in community of Gayasan National Park in Korea (Continued)

\begin{tabular}{|c|c|c|c|c|c|c|c|c|c|}
\hline Boraginaceae & $\begin{array}{l}\text { Lithospermum } \\
\text { erythrorhizon Siebold \& } \\
\text { Zucc. }\end{array}$ & KH5379 & S102 & Jichi & Root & Bruising & Infusion, poultice & Oral, topical & 10.00 \\
\hline & & & & & & Convulsion & $\begin{array}{l}\text { Decoction, infusion, oil, panbroiled, poultice, } \\
\text { roast }\end{array}$ & Oral, topical & 83.33 \\
\hline & & & & & & Indigestion & Panbroiled & Oral & 6.67 \\
\hline \multirow[t]{6}{*}{ Brassicaceae } & $\begin{array}{l}\text { Brassica rapa var. glabra } \\
\text { Regel }\end{array}$ & KH5380 & S30 & Baechu & Leaf & Burn & Kimchi, raw & Topical & 100.00 \\
\hline & $\begin{array}{l}\text { Capsella bursapastoris (L.) } \\
\text { L.W.Medicus }\end{array}$ & KH5381 & S34 & Naengi & Whole part & Liver diseases & Juice, seasoned cooked vegetables, soup & Oral & 100.00 \\
\hline & Raphanus sativus L. & KH5382 & S151 & $\mathrm{Mu}$ & Leaf & Lumbago & Steamed & Topical & 1.72 \\
\hline & & & & & $\begin{array}{l}\text { Tuberous } \\
\text { root }\end{array}$ & Bronchitis & Infusion & Oral & 4.31 \\
\hline & & & & & & Common cold & $\begin{array}{l}\text { Decoction, extraction, fermentation, infusion, } \\
\text { raw, roast }\end{array}$ & Oral & 56.90 \\
\hline & & & & & & Cough & Infusion, raw, roast & Oral & 37.07 \\
\hline \multirow[t]{15}{*}{ Campanulaceae } & $\begin{array}{l}\text { Adenophora triphylla var. } \\
\text { japonica (Regel) H. Hara }\end{array}$ & KH5383 & S6 & Jandae & Root & Sterility & Infusion & Oral & 20.00 \\
\hline & & & & & & $\begin{array}{l}\text { Woman } \\
\text { diseases }\end{array}$ & $\begin{array}{l}\text { A sweet drink made from fermented rice, } \\
\text { raw, simmer }\end{array}$ & Oral & 80.00 \\
\hline & $\begin{array}{l}\text { Codonopsis lanceolata } \\
\text { (Siebold \& Zucc.) Trautv. }\end{array}$ & KH5384 & S50 & Deodeok & Root & $\begin{array}{l}\text { Abdominal } \\
\text { cold } \\
\text { hypersentivity }\end{array}$ & Maceration, raw & Oral & 17.39 \\
\hline & & & & & & Bronchitis & Maceration, raw & Oral & 19.57 \\
\hline & & & & & & Cough & Steamed & Oral & 10.87 \\
\hline & & & & & & Orchiopathy & Brewing, maceration, raw & Oral & 26.09 \\
\hline & & & & & & $\begin{array}{l}\text { Sexual } \\
\text { enhancement }\end{array}$ & Brewing, infusion & Oral & 26.09 \\
\hline & $\begin{array}{l}\text { Codonopsis ussuriensis } \\
\text { (Rupr. \& Maxim.) Hemsl. }\end{array}$ & KH5385 & S51 & Sogyeongbulal & Root & $\begin{array}{l}\text { Sexual } \\
\text { enhancement }\end{array}$ & Infusion & Oral & 100.00 \\
\hline & $\begin{array}{l}\text { Platycodon grandiflorum } \\
\text { (Jacq.) A.DC. }\end{array}$ & KH5386 & S134 & Doraji & Flower & Sterility & Infusion & Oral & 2.62 \\
\hline & & & & & Root & $\begin{array}{l}\text { Abdominal } \\
\text { pain }\end{array}$ & Infusion & Oral & 1.12 \\
\hline & & & & & & Anorexia & Infusion & Oral & 1.12 \\
\hline & & & & & & Asthma & Brewing & Oral & 11.99 \\
\hline & & & & & & & Dried, fermentation, infusion, powder, pill & Oral & \\
\hline & & & & & & Bronchitis & Extraction, infusion, juice, powder & Oral & 7.12 \\
\hline & & & & & & Common cold & $\begin{array}{l}\text { Brewing, decoction, dried, infusion, } \\
\text { maceration, mixed in honey, pill, powder }\end{array}$ & Oral & 21.72 \\
\hline
\end{tabular}


Table 2 Plant species for medicinal purposes in community of Gayasan National Park in Korea (Continued)

\begin{tabular}{|c|c|c|c|c|c|c|c|c|c|}
\hline & & & & & & Cough & $\begin{array}{l}\text { Brewing, decoction, dissolution, dried, } \\
\text { extraction, fermentation, infusion, } \\
\text { maceration, mixed in honey, powder, pill }\end{array}$ & Oral & 50.56 \\
\hline & & & & & & Hyperthermia & Infusion & Oral & 1.50 \\
\hline & & & & & & Leukorrhea & Infusion & Oral & 1.50 \\
\hline & & & & & & $\begin{array}{l}\text { Sinews and } \\
\text { joint pain }\end{array}$ & Infusion & Oral & 0.75 \\
\hline & & & & & & Sterility & Infusion & Oral & 2.62 \\
\hline \multirow[t]{2}{*}{ Cannabaceae } & Cannabis sativa $\mathrm{L}$. & KH5387 & $\mathrm{S} 33$ & Sam & Leaf & Paralysis & Infusion & Oral & 100.00 \\
\hline & $\begin{array}{l}\text { Humulus japonicus } \\
\text { Sieboid \& Zucc. }\end{array}$ & KH5388 & 590 & Hwansamdeonggul & Aerial part & Athlete's foot & Juice & Topical & 100.00 \\
\hline \multirow[t]{10}{*}{ Caprifoliaceae } & Lonicera japonica Thunb. & KH5389 & S103 & Indongdeonggul & Aerial part & Bone diseases & A sweet drink made from fermented rice & Oral & 36.11 \\
\hline & & & & & Flower & $\begin{array}{l}\text { Lack of } \\
\text { energy }\end{array}$ & Brewing & Oral & 1.39 \\
\hline & & & & & Stem & Arm pain & $\begin{array}{l}\text { A sweet drink made from fermented rice, } \\
\text { brewing, decoction, infusion }\end{array}$ & Oral & 19.79 \\
\hline & & & & & & Bone diseases & $\begin{array}{l}\text { A sweet drink made from fermented rice, } \\
\text { brewing, decoction, infusion }\end{array}$ & Oral & 36.11 \\
\hline & & & & & & Bronchitis & Infusion & Oral & 0.35 \\
\hline & & & & & & $\begin{array}{l}\text { Carpal tunnel } \\
\text { syndrome }\end{array}$ & Infusion & Oral & 1.04 \\
\hline & & & & & & $\begin{array}{l}\text { Lack of } \\
\text { energy }\end{array}$ & A sweet drink made from fermented rice & Oral & 1.39 \\
\hline & & & & & & Leg pain & $\begin{array}{l}\text { A sweet drink made from fermented rice } \\
\text { brewing, decoction, infusion }\end{array}$ & Oral & 26.04 \\
\hline & & & & & & Lumbago & $\begin{array}{l}\text { A sweet drink made from fermented rice, } \\
\text { infusion }\end{array}$ & Oral & 14.24 \\
\hline & & & & & & $\begin{array}{l}\text { Tarsal tunnel } \\
\text { syndrome }\end{array}$ & Infusion & Oral & 1.04 \\
\hline Caryophyllaceae & Dianthus longicalyx Miq. & KH5390 & $\mathrm{S} 60$ & Sulpaeraengikkot & Whole part & Pollakiuria & Infusion & Oral & 100.00 \\
\hline \multirow[t]{4}{*}{ Celastraceae } & $\begin{array}{l}\text { Euonymus alatus (Thunb.) } \\
\text { Siebold }\end{array}$ & KH5391 & $\mathrm{S} 70$ & Hwasalnamu & Leaf & $\begin{array}{l}\text { Gastroenteric } \\
\text { disorder }\end{array}$ & Seasoned cooked vegetables & Oral & 100.00 \\
\hline & & & & & Stem & $\begin{array}{l}\text { Gastroenteric } \\
\text { disorder }\end{array}$ & Decoction & Oral & 100.00 \\
\hline & $\begin{array}{l}\text { Euonymus sachalinensis ( } \mathrm{F} \text {. } \\
\text { Schmidt) Maxim. }\end{array}$ & KH5392 & $\mathrm{S} 71$ & Hoenamu & Leaf & Hemorrhoids & Infusion & Topical & 100.00 \\
\hline & & & & & Stem & Hemorrhoids & Fumigation, infusion & Topical & 100.00 \\
\hline Chenopodiaceae & $\begin{array}{l}\text { Kochia scoparia (L.) } \\
\text { Schrad. var. scoparia }\end{array}$ & KH5393 & 596 & Daepssari & Aerial part & Pollakiuria & Infusion & Oral & 100.00 \\
\hline
\end{tabular}


Table 2 Plant species for medicinal purposes in community of Gayasan National Park in Korea (Continued)

\begin{tabular}{|c|c|c|c|c|c|c|c|c|c|}
\hline Commelinaceae & Commelina communis L. & KH5394 & $\mathrm{S} 53$ & Dalkuijangpul & Whole part & Bruising & Maceration & Topical & 33.33 \\
\hline & & & & & & Snakebite & Maceration & Topical & 33.33 \\
\hline & & & & & & Sprain & Maceration & Topical & 33.33 \\
\hline Convolvulaceae & Ipomoea batatas (L.) Lam. & KH5395 & 593 & Goguma & Root & Pig disease & Raw & Oral & 100.00 \\
\hline \multirow[t]{2}{*}{ Cornaceae } & $\begin{array}{l}\text { Cornus officinalis Siebold } \\
\text { \& Zucc. }\end{array}$ & KH5396 & S55 & Sansuyu & Fruit & $\begin{array}{l}\text { Lack of } \\
\text { energy }\end{array}$ & Decoction & Oral & 53.85 \\
\hline & & & & & & Pollakiuria & Infusion & Oral & 46.15 \\
\hline \multirow[t]{7}{*}{ Crassulaceae } & $\begin{array}{l}\text { Orostachys japonica } \\
\text { (Maxim.) A.Berger }\end{array}$ & KH5397 & S112 & Bawisol & Aerial part & Cancer & Maceration & Oral & 75.00 \\
\hline & & & & & Whole part & Cancer & Dried, infusion & Oral & 75.00 \\
\hline & & & & & & Snakebite & Maceration & Topical & 25.00 \\
\hline & $\begin{array}{l}\text { Sedum sarmentosum } \\
\text { Bunge }\end{array}$ & KH5398 & S171 & Dolnamul & Whole part & $\begin{array}{l}\text { Gastroenteric } \\
\text { disorder }\end{array}$ & Watery plain Kimchi & Oral & 13.21 \\
\hline & & & & & & Hepatitis & Juice & Oral & 16.98 \\
\hline & & & & & & Liver diseases & Maceration, powder, raw, watery plain Kimchi & Oral & 52.83 \\
\hline & & & & & & Snakebite & Maceration, raw & Topical & 16.98 \\
\hline \multirow[t]{16}{*}{ Cucurbitaceae } & Citrullus vulgaris Schrad. & KH5399 & S46 & Subak & Fruit & Overheating & Extraction & Oral & 100.00 \\
\hline & $\begin{array}{l}\text { Cucumis melo var. } \\
\text { makuwa Makino }\end{array}$ & KH5400 & S56 & Chamoe & Peduncle & Jaundice & Powder & Topical & 55.56 \\
\hline & & & & & & Otalgia & Powder & Topical & 44.44 \\
\hline & Cucumis sativus $\mathrm{L}$. & KH5401 & $\mathrm{S} 57$ & Oi & Stem & Pollakiuria & Dried, infusion & Oral & 100.00 \\
\hline & $\begin{array}{l}\text { Cucurbita moschata } \\
\text { Duchesne }\end{array}$ & KH5402 & S58 & Hobak & Fruit & Asthma & Simmer & Oral & 11.11 \\
\hline & & & & & & Edema & Infusion, roast & Oral & 61.11 \\
\hline & & & & & & $\begin{array}{l}\text { Gastroenteric } \\
\text { disorder }\end{array}$ & Infusion & Oral & 27.78 \\
\hline & $\begin{array}{l}\text { Lagenaria leucantha } \\
\text { Rusby }\end{array}$ & KH5403 & S98 & Bak & Endodermis & Cattle disease & Infusion & Oral & 56.25 \\
\hline & & & & & Fruit & Cattle disease & Infusion & Oral & 56.25 \\
\hline & & & & & & Common cold & Infusion & Oral & 31.25 \\
\hline & & & & & Pericarp & Pollakiuria & Infusion & Oral & 12.50 \\
\hline & Luffa cylindrica Roem. & KH5404 & $\mathrm{S} 104$ & Susemioi & Fruit & Asthma & Extraction, sap & Oral & 19.51 \\
\hline & & & & & & Bronchitis & Extraction & Oral & 10.98 \\
\hline & & & & & & $\begin{array}{l}\text { Chronic } \\
\text { cough }\end{array}$ & Extraction, sap & Oral & 4.88 \\
\hline & & & & & & Cough & $\begin{array}{l}\text { A sweet drink made from fermented rice, } \\
\text { extraction, fermentation, infusion, oil, pan } \\
\text { fried, sap }\end{array}$ & Oral & 43.90 \\
\hline & & & & & & Skin diseases & Extraction, sap & Oral, Topical & 15.85 \\
\hline
\end{tabular}


Table 2 Plant species for medicinal purposes in community of Gayasan National Park in Korea (Continued)

\begin{tabular}{|c|c|c|c|c|c|c|c|c|c|}
\hline & & & & & Stem & Asthma & Sap & Oral & 19.51 \\
\hline & & & & & & $\begin{array}{l}\text { Atopic } \\
\text { dermatitis }\end{array}$ & Raw & Topical & 4.88 \\
\hline & & & & & & Bronchitis & Sap & Oral & 10.98 \\
\hline & & & & & & Skin diseases & Raw & Topical & 15.85 \\
\hline & $\begin{array}{l}\text { Trichosanthes kirilowii } \\
\text { Maxim. }\end{array}$ & KH5405 & S186 & Haneultari & Fruit & Bruising & Dough & Topical & 4.69 \\
\hline & & & & & & $\begin{array}{l}\text { Carpal tunnel } \\
\text { syndrome }\end{array}$ & Dough & Topical & 1.56 \\
\hline & & & & & & $\begin{array}{l}\text { Chronic } \\
\text { myofascial } \\
\text { pain }\end{array}$ & Brewing, infusion & Oral & 37.50 \\
\hline & & & & & & Lumbago & Brewing, infusion & Oral & 12.50 \\
\hline & & & & & & Neuralgia & Infusion & Oral & 9.38 \\
\hline & & & & & & Overheating & Extraction, infusion & Oral & 18.75 \\
\hline & & & & & & $\begin{array}{l}\text { Tarsal tunnel } \\
\text { syndrome }\end{array}$ & Dough & Topical & 1.56 \\
\hline & & & & & & Urticaria & Infusion & Oral & 9.38 \\
\hline & & & & & Root & $\begin{array}{l}\text { Induced } \\
\text { abortion }\end{array}$ & Maceration & Oral & 4.69 \\
\hline & & & & & & Neuralgia & Infusion & Oral & 9.38 \\
\hline & & & & & & Overheating & Infusion & Oral & 18.75 \\
\hline & & & & & Stem & Neuralgia & Infusion & Oral & 9.38 \\
\hline \multirow[t]{5}{*}{ Dioscoreaceae } & Dioscorea batatas Decne. & KH5406 & $\mathrm{S} 61$ & Ma & Root & $\begin{array}{l}\text { Gastroenteric } \\
\text { disorder }\end{array}$ & Maceration, oil, raw & Oral & 37.14 \\
\hline & & & & & & $\begin{array}{l}\text { Lack of } \\
\text { energy }\end{array}$ & Maceration, oil, raw & Oral & 34.29 \\
\hline & & & & & & Milk fever & Maceration & Oral, topical & 17.14 \\
\hline & & & & & & Osteoporosis & Maceration & Oral & 11.43 \\
\hline & Dioscorea japonica Thunb. & KH5407 & $\mathrm{S} 62$ & Chamma & Root & $\begin{array}{l}\text { Gastroenteric } \\
\text { disorder }\end{array}$ & Raw & Oral & 100.00 \\
\hline \multirow[t]{7}{*}{ Ebenaceae } & Diospyros kaki Thunb. & KH5408 & $\mathrm{S} 63$ & Gamnamu & Fruit & Boils & Raw & Topical & 4.05 \\
\hline & & & & & & Cough & Ripe persimmon & Oral & 6.76 \\
\hline & & & & & & Diarrhea & Raw, roast & Oral & 9.46 \\
\hline & & & & & & Indigestion & Infusion, raw & Oral & 47.30 \\
\hline & & & & & Peduncle & $\begin{array}{l}\text { Acute } \\
\text { gastroenteritis }\end{array}$ & Infusion & Oral & 6.76 \\
\hline & & & & & & Hiccups & Decoction, infusion & Oral & 18.92 \\
\hline & & & & & & Indigestion & Infusion & Oral & 47.30 \\
\hline
\end{tabular}


Table 2 Plant species for medicinal purposes in community of Gayasan National Park in Korea (Continued)

\begin{tabular}{|c|c|c|c|c|c|c|c|c|c|}
\hline & & & & & Pericarp & Common cold & Infusion & Oral & 6.76 \\
\hline \multirow[t]{7}{*}{ Elaeagnaceae } & $\begin{array}{l}\text { Elaeagnus umbellata } \\
\text { Thunb. }\end{array}$ & KH5409 & $\mathrm{S} 65$ & Borisunamu & Fruit & Asthma & Raw & Oral & 11.59 \\
\hline & & & & & & Bronchitis & Extraction & Oral & 4.35 \\
\hline & & & & & & Common cold & Extraction & Oral & 5.80 \\
\hline & & & & & & Cough & $\begin{array}{l}\text { A sweet drink made from fermented rice, } \\
\text { brewing, extraction, raw }\end{array}$ & Oral & 75.36 \\
\hline & & & & & Stem & Asthma & A sweet drink made from fermented rice & Oral & 11.59 \\
\hline & & & & & & Cough & $\begin{array}{l}\text { A sweet drink made from fermented rice, } \\
\text { infusion }\end{array}$ & Oral & 75.36 \\
\hline & & & & & & Lumbago & A sweet drink made from fermented rice & Oral & 2.90 \\
\hline \multirow[t]{2}{*}{ Ericaceae } & $\begin{array}{l}\text { Rhododendron } \\
\text { mucronulatum Turcz. }\end{array}$ & KH5410 & S153 & Jindalrae & Stem & Bone diseases & A sweet drink made from fermented rice & Oral & 100.00 \\
\hline & $\begin{array}{l}\text { Rhododendron weyrichii } \\
\text { Maxim. }\end{array}$ & KH5411 & S154 & Chamkkoknamu & Root & Cough & Extraction & Oral & 100.00 \\
\hline \multirow[t]{5}{*}{ Eucommiaceae } & Eucommia ulmoides Oliv. & KH5412 & S69 & Duchung & Bark & Arm pain & A sweet drink made from fermented rice & Oral & 21.05 \\
\hline & & & & & & Bone diseases & A sweet drink made from fermented rice & Oral & 21.05 \\
\hline & & & & & & Cancer & Infusion & Oral & 10.53 \\
\hline & & & & & & Leg pain & A sweet drink made from fermented rice & Oral & 21.05 \\
\hline & & & & & & Lumbago & $\begin{array}{l}\text { A sweet drink made from fermented rice, } \\
\text { decoction }\end{array}$ & Oral & 26.32 \\
\hline \multirow[t]{7}{*}{ Euphorbiaceae } & Ricinus communis $\mathrm{L}$. & KH5413 & S157 & Pimaja & Aerial part & Lumbago & A sweet drink made from fermented rice & Oral & 2.80 \\
\hline & & & & & Fruit & Constipation & Oil, pan fried & Oral, topical & 42.66 \\
\hline & & & & & & Cough & Oil, pan fried & Oral & 2.80 \\
\hline & & & & & & Dental pain & Roast & Topical & 3.50 \\
\hline & & & & & & Indigestion & Oil, pan fried & Oral & 44.06 \\
\hline & & & & & & Pig disease & Oil & Oral & 1.40 \\
\hline & & & & & Leaf & Otalgia & Infusion & Topical & 2.80 \\
\hline \multirow[t]{8}{*}{ Fabaceae } & Albizia julibrissin Durazz. & KH5414 & S8 & Jagwinamu & Stem & Arm pain & A sweet drink made from fermented rice & Oral & 18.18 \\
\hline & & & & & & Bone diseases & $\begin{array}{l}\text { A sweet drink made from fermented rice, } \\
\text { brewing }\end{array}$ & Oral & 54.55 \\
\hline & & & & & & Leg pain & A sweet drink made from fermented rice & Oral & 18.18 \\
\hline & & & & & & Neuralgia & Infusion & Oral & 9.09 \\
\hline & $\begin{array}{l}\text { Astragalus mongholicus } \\
\text { Bunge }\end{array}$ & KH5415 & S27 & Hwanggi & Root & Afterpain & Decoction, infusion, simmer & Oral & 25.00 \\
\hline & & & & & & Bone diseases & A sweet drink made from fermented rice & Oral & 15.63 \\
\hline & & & & & & Hyperridrosis & Boiling, infusion & Oral & 37.50 \\
\hline & & & & & & Lumbago & Simmer & Oral & 15.63 \\
\hline
\end{tabular}


Table 2 Plant species for medicinal purposes in community of Gayasan National Park in Korea (Continued)

\begin{tabular}{|c|c|c|c|c|c|c|c|c|}
\hline & & & & & $\begin{array}{l}\text { Woman } \\
\text { diseases }\end{array}$ & Simmer & Oral & 6.25 \\
\hline $\begin{array}{l}\text { Caragana sinica (Buc'hoz) } \\
\text { Rehder }\end{array}$ & KH5416 & S36 & Goldamcho & Leaf & $\begin{array}{l}\text { Chronic } \\
\text { myofascial } \\
\text { pain }\end{array}$ & Brewing, maceration & Oral, topical & 9.00 \\
\hline & & & & & Leg pain & Brewing, maceration & Oral, topical & 18.51 \\
\hline & & & & & Arm pain & $\begin{array}{l}\text { A sweet drink made from fermented rice, } \\
\text { brewing, infusion }\end{array}$ & Oral & 12.85 \\
\hline & & & & & Arthritis & Infusion & Oral & 1.54 \\
\hline & & & & & Bone diseases & $\begin{array}{l}\text { A sweet drink made from fermented rice, } \\
\text { brewing, infusion, juice }\end{array}$ & Oral & 47.56 \\
\hline & & & & & $\begin{array}{l}\text { Chronic } \\
\text { myofascial } \\
\text { pain }\end{array}$ & Brewing, juice, maceration & Oral, topical & 9.00 \\
\hline & & & & & $\begin{array}{l}\text { Gastroenteric } \\
\text { disorder }\end{array}$ & A sweet drink made from fermented rice & Oral & 1.03 \\
\hline & & & & & Leg pain & $\begin{array}{l}\text { A sweet drink made from fermented rice, } \\
\text { brewing, infusion, maceration }\end{array}$ & Oral, topical & 18.51 \\
\hline & & & & & Lumbago & $\begin{array}{l}\text { A sweet drink made from fermented rice, } \\
\text { infusion, juice }\end{array}$ & Oral & 8.48 \\
\hline & & & & & Neuralgia & $\begin{array}{l}\text { A sweet drink made from fermented rice, } \\
\text { brewing, infusion }\end{array}$ & Oral & 1.03 \\
\hline Gleditsia sinensis Lamarck & KH5417 & $\mathrm{S} 83$ & Jogakjanamu & Stem & Arm pain & A sweet drink made from fermented rice & Oral & 26.92 \\
\hline & & & & & Bone diseases & A sweet drink made from fermented rice & Oral & 11.54 \\
\hline & & & & & Leg pain & A sweet drink made from fermented rice & Oral & 26.92 \\
\hline & & & & & Lumbago & $\begin{array}{l}\text { A sweet drink made from fermented rice, } \\
\text { infusion }\end{array}$ & Oral & 19.23 \\
\hline & & & & & Shoulder pain & A sweet drink made from fermented rice & Oral & 15.38 \\
\hline Glycine max (L.) Merr. & KH5418 & S84 & Kong & Seed & $\begin{array}{l}\text { Acute } \\
\text { gastroenteritis }\end{array}$ & Raw & Oral & 3.33 \\
\hline & & & & & Asthma & Oil & Oral & 1.43 \\
\hline & & & & & Bee sting & Fermentation & Topical & 0.95 \\
\hline & & & & & $\begin{array}{l}\text { Carpal tunnel } \\
\text { syndrome }\end{array}$ & $\begin{array}{l}\text { A sweet drink made from fermented rice, } \\
\text { infusion }\end{array}$ & Oral & 3.81 \\
\hline & & & & & Common cold & Fermentation, roast & Oral & 23.33 \\
\hline & & & & & Cough & Fermentation, infusion, oil, panbroiled, roast & Oral & 19.05 \\
\hline & & & & & Hangover & Soup & Oral & 0.48 \\
\hline & & & & & $\begin{array}{l}\text { Head } \\
\text { laceration }\end{array}$ & Fermentation & Topical & 17.14 \\
\hline & & & & & Hemorrhaging & Boiling, fermentation, fumigation & Topical & 5.24 \\
\hline
\end{tabular}


Table 2 Plant species for medicinal purposes in community of Gayasan National Park in Korea (Continued)

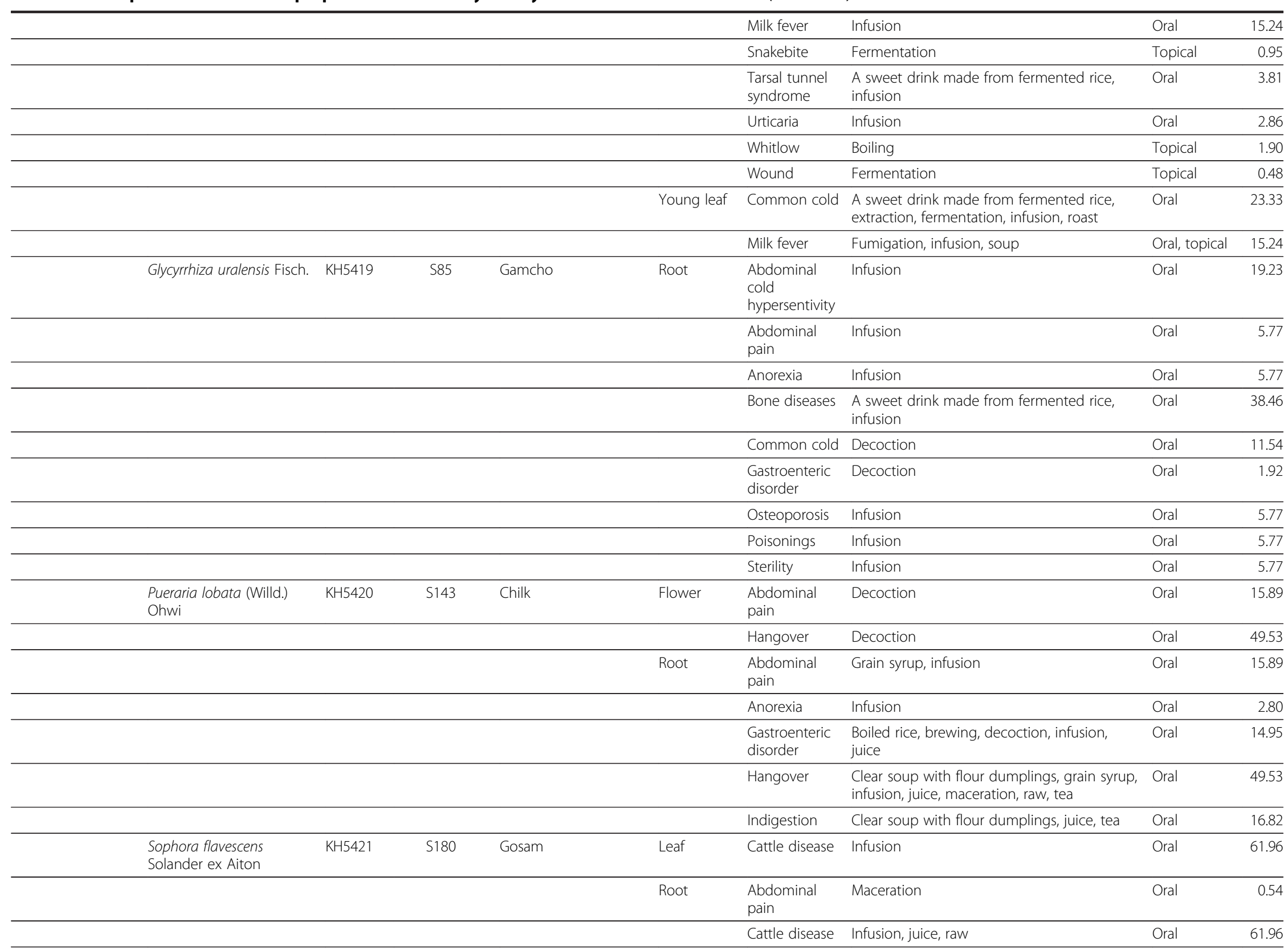


Table 2 Plant species for medicinal purposes in community of Gayasan National Park in Korea (Continued)

\begin{tabular}{|c|c|c|c|c|c|c|c|c|c|}
\hline & & & & & & $\begin{array}{l}\text { Chronic } \\
\text { myofascial } \\
\text { pain }\end{array}$ & Dissolution & Oral & 2.17 \\
\hline & & & & & & $\begin{array}{l}\text { Gastroenteric } \\
\text { disorder }\end{array}$ & Infusion & Oral & 3.80 \\
\hline & & & & & & $\begin{array}{l}\text { Hansen's } \\
\text { Disease }\end{array}$ & Dried, infusion & Oral & 3.80 \\
\hline & & & & & & Leg pain & Infusion, maceration & Oral & 5.43 \\
\hline & & & & & & Lumbago & Juice, maceration & Oral & 7.61 \\
\hline & & & & & & Neuralgia & Infusion, juice & Oral & 5.43 \\
\hline & & & & & & Paralysis & Infusion & Oral & 2.72 \\
\hline & & & & & & Pruritus & Dried, maceration, mixed in liquor, pill & Oral & 1.63 \\
\hline & & & & & & Pus & Maceration & Topical & 2.72 \\
\hline & & & & & & Skin diseases & Maceration, raw & Oral & 2.17 \\
\hline & $\begin{array}{l}\text { Vigna angularis (Willd.) } \\
\text { Ohwi \& H.Ohashi }\end{array}$ & KH5422 & S190 & Pat & Seed & Cattle disease & Infusion & Oral & 100.00 \\
\hline \multirow[t]{10}{*}{ Fagaceae } & $\begin{array}{l}\text { Castanea crenata Siebold } \\
\& \text { Zucc. }\end{array}$ & KH5423 & S38 & Bamnamu & Bark & Indigestion & Infusion & Oral & 6.02 \\
\hline & & & & & & $\begin{array}{l}\text { Lacquer } \\
\text { poisoning }\end{array}$ & Infusion & Topical & 27.71 \\
\hline & & & & & Fruit & $\begin{array}{l}\text { Abdominal } \\
\text { cold } \\
\text { hypersentivity }\end{array}$ & Decoction, grain syrup, infusion, pill, simmer & Oral & 36.14 \\
\hline & & & & & & Anorexia & Simmer, pill & Oral & 6.02 \\
\hline & & & & & & $\begin{array}{l}\text { Lack of } \\
\text { energy }\end{array}$ & Simmer, pill & Oral & 6.02 \\
\hline & & & & & & Measles & Panbroiled & Oral & 3.61 \\
\hline & & & & & & Sterility & Grain syrup, infusion, Simmer, pill & Oral & 14.46 \\
\hline & & & & & Leaf & $\begin{array}{l}\text { Lacquer } \\
\text { poisoning }\end{array}$ & Infusion & Topical & 27.71 \\
\hline & & & & & Stem & $\begin{array}{l}\text { Lacquer } \\
\text { poisoning }\end{array}$ & Infusion & Topical & 27.71 \\
\hline & $\begin{array}{l}\text { Quercus acutissima } \\
\text { Carruth. }\end{array}$ & KH5424 & $\mathrm{S} 148$ & Sangsurinamu & Fruit & Cattle disease & Raw & Oral & 100.00 \\
\hline \multirow[t]{5}{*}{ Ganodermataceae } & $\begin{array}{l}\text { Ganoderma lucidum } \\
\text { (Curtis) P. Karst. }\end{array}$ & KH5425 & S77 & Bulrocho & Whole part & $\begin{array}{l}\text { Abdominal } \\
\text { pain }\end{array}$ & Boiling & Oral & 4.35 \\
\hline & & & & & & Bone diseases & A sweet drink made from fermented rice & Oral & 13.04 \\
\hline & & & & & & Cancer & Boiling, decoction & Oral & 26.09 \\
\hline & & & & & & Common cold & Boiling & Oral & 26.09 \\
\hline & & & & & & Indigestion & Infusion & Oral & 13.04 \\
\hline
\end{tabular}


Table 2 Plant species for medicinal purposes in community of Gayasan National Park in Korea (Continued)

\begin{tabular}{|c|c|c|c|c|c|c|c|c|c|}
\hline & & & & & & $\begin{array}{l}\text { Woman } \\
\text { diseases }\end{array}$ & Dried, infusion & Oral & 17.39 \\
\hline \multirow[t]{2}{*}{ Gentianaceae } & Gentiana scabra Bunge & KH5426 & 580 & Yongdam & Leaf & Pollakiuria & Infusion & Oral & 45.45 \\
\hline & & & & & Root & $\begin{array}{l}\text { Abdominal } \\
\text { pain }\end{array}$ & $\begin{array}{l}\text { A sweet drink made from fermented rice, } \\
\text { infusion }\end{array}$ & Oral & 54.55 \\
\hline \multirow[t]{2}{*}{ Geraniaceae } & $\begin{array}{l}\text { Geranium thunbergii } \\
\text { Siebold \& Zucc. }\end{array}$ & KH5427 & S81 & ljilpul & Leaf & $\begin{array}{l}\text { Abdominal } \\
\text { pain }\end{array}$ & Infusion & Oral & 100.00 \\
\hline & & & & & Whole part & $\begin{array}{l}\text { Abdominal } \\
\text { pain }\end{array}$ & Infusion & Oral & 100.00 \\
\hline \multirow[t]{2}{*}{ Ginkgoaceae } & Ginkgo biloba L. & KH5428 & 582 & Eunhaengnamu & Fruit & Asthma & Brewing & Oral & 33.33 \\
\hline & & & & & & Glycosuria & Infusion, roast & Oral & 66.67 \\
\hline \multirow[t]{4}{*}{ Hymenochaetaceae } & $\begin{array}{l}\text { Phellinus linteus (Berk. \& } \\
\text { Curt.) Teng }\end{array}$ & KH5429 & S124 & Mokjiljinheulkbeoseot & Whole part & Cancer & Boiling, infusion & Oral & 68.97 \\
\hline & & & & & & $\begin{array}{l}\text { Gastroenteric } \\
\text { disorder }\end{array}$ & Infusion & Oral & 10.34 \\
\hline & & & & & & Hypofunction & Infusion & Oral & 3.45 \\
\hline & & & & & & Lumbago & Infusion & Oral & 17.24 \\
\hline \multirow[t]{10}{*}{ Juglandaceae } & Juglans regia L. & KH5430 & S94 & Hodunamu & Fruit & $\begin{array}{l}\text { Lack of } \\
\text { energy }\end{array}$ & Raw & Oral & 100.00 \\
\hline & $\begin{array}{l}\text { Platycarya strobilacea } \\
\text { Siebold \& Zucc. }\end{array}$ & KH5431 & $S 133$ & Gulpinamu & Stem & $\begin{array}{l}\text { Abdominal } \\
\text { pain }\end{array}$ & Infusion & Oral & 1.27 \\
\hline & & & & & & Anorexia & Infusion & Oral & 0.76 \\
\hline & & & & & & Arm pain & $\begin{array}{l}\text { A sweet drink made from fermented rice, } \\
\text { brewing, decoction }\end{array}$ & Oral & 6.36 \\
\hline & & & & & & Bone diseases & $\begin{array}{l}\text { A sweet drink made from fermented rice, } \\
\text { brewing, decoction, infusion }\end{array}$ & Oral & 60.31 \\
\hline & & & & & & Cattle disease & Infusion & Oral & 3.82 \\
\hline & & & & & & Hyperthermia & $\begin{array}{l}\text { A sweet drink made from fermented rice, } \\
\text { brewing }\end{array}$ & Oral & 0.51 \\
\hline & & & & & & Leg pain & $\begin{array}{l}\text { A sweet drink made from fermented rice, } \\
\text { brewing, decoction, infusion }\end{array}$ & Oral & 16.03 \\
\hline & & & & & & Lumbago & $\begin{array}{l}\text { A sweet drink made from fermented rice, } \\
\text { infusion }\end{array}$ & Oral & 10.43 \\
\hline & & & & & & Neuralgia & Infusion & Oral & 0.51 \\
\hline \multirow[t]{3}{*}{ Lamiaceae } & Leonurus japonicus Houtt. & KH5432 & S100 & Ikmocho & Aerial part & $\begin{array}{l}\text { Abdominal } \\
\text { cold } \\
\text { hypersentivity }\end{array}$ & $\begin{array}{l}\text { A sweet drink made from fermented rice, } \\
\text { decoction, dried, grain syrup, infusion, juice, } \\
\text { maceration, pill, raw, simmer, taffy }\end{array}$ & Oral & 42.17 \\
\hline & & & & & & $\begin{array}{l}\text { Abdominal } \\
\text { pain }\end{array}$ & Infusion, juice & Oral & 2.07 \\
\hline & & & & & & Afterpain & Maceration & Oral & 1.61 \\
\hline
\end{tabular}


Table 2 Plant species for medicinal purposes in community of Gayasan National Park in Korea (Continued)

\begin{tabular}{|c|c|c|c|c|}
\hline & Anorexia & Maceration, pill, simmer & Oral & 3.00 \\
\hline & Bone diseases & $\begin{array}{l}\text { A sweet drink made from fermented rice, } \\
\text { dried, infusion }\end{array}$ & Oral & 0.92 \\
\hline & Cattle disease & Brewing, maceration & Oral & 1.38 \\
\hline & Dysmenorrhea & Infusion & Oral & 1.61 \\
\hline & Fatigue & Maceration & Oral & 1.38 \\
\hline & Jaundice & $\begin{array}{l}\text { A sweet drink made from fermented rice, } \\
\text { infusion, juice, pill, tea }\end{array}$ & Oral & 1.84 \\
\hline & $\begin{array}{l}\text { Lack of } \\
\text { energy }\end{array}$ & $\begin{array}{l}\text { A sweet drink made from fermented rice, } \\
\text { dried, infusion, pill, simmer }\end{array}$ & Oral & 2.07 \\
\hline & Leukorrhea & Infusion & Oral & 2.53 \\
\hline & Liver diseases & Infusion, pill & Oral & 0.46 \\
\hline & $\begin{array}{l}\text { Raynaud's } \\
\text { Phenomenon }\end{array}$ & $\begin{array}{l}\text { A sweet drink made from fermented rice, } \\
\text { infusion, juice }\end{array}$ & Oral & 4.84 \\
\hline & Sterility & $\begin{array}{l}\text { A sweet drink made from fermented rice, } \\
\text { decoction, infusion, juice, maceration, pill, } \\
\text { simmer }\end{array}$ & Oral & 21.20 \\
\hline & $\begin{array}{l}\text { Woman } \\
\text { diseases }\end{array}$ & $\begin{array}{l}\text { Boiling dried, grain syrup, infusion, juice, pill, } \\
\text { simmer, taffy }\end{array}$ & Oral & 10.60 \\
\hline Leaf & $\begin{array}{l}\text { Abdominal } \\
\text { cold } \\
\text { hypersentivity }\end{array}$ & Grain syrup, infusion, pill & Oral & 42.17 \\
\hline & Anorexia & Juice & Oral & 3.00 \\
\hline & $\begin{array}{l}\text { Irregular } \\
\text { menstruation }\end{array}$ & Infusion & Oral & 0.46 \\
\hline & $\begin{array}{l}\text { Raynaud's } \\
\text { Phenomenon }\end{array}$ & Infusion & Oral & 4.84 \\
\hline & Sterility & Grain syrup, infusion, simmer & Oral & 21.20 \\
\hline & $\begin{array}{l}\text { Woman } \\
\text { diseases }\end{array}$ & Grain syrup & Oral & 10.60 \\
\hline Root & Sterility & Infusion & Oral & 21.20 \\
\hline Stem & $\begin{array}{l}\text { Raynaud's } \\
\text { Phenomenon }\end{array}$ & Juice & Oral & 4.84 \\
\hline & Sterility & Grain syrup, juice & Oral & 21.20 \\
\hline Whole part & $\begin{array}{l}\text { Abdominal } \\
\text { cold } \\
\text { hypersentivity }\end{array}$ & Decoction, grain syrup, infusion, juice & Oral & 42.17 \\
\hline & Paralysis & Decoction, juice & Oral & 0.92 \\
\hline & Sterility & Grain syrup, juice & Oral & 21.20 \\
\hline & Overheating & Juice & Oral & 0.92 \\
\hline
\end{tabular}


Table 2 Plant species for medicinal purposes in community of Gayasan National Park in Korea (Continued)

\begin{tabular}{|c|c|c|c|c|c|c|c|c|}
\hline & & & & & $\begin{array}{l}\text { Woman } \\
\text { diseases }\end{array}$ & Decoction, juice & Oral & 10.60 \\
\hline $\begin{array}{l}\text { Mentha piperascens } \\
\text { (Malinv.) Holmes }\end{array}$ & KH5433 & S107 & Bakha & Stem & Dental pain & Maceration & Topical & 100.00 \\
\hline $\begin{array}{l}\text { Perilla frutescens var. acuta } \\
\text { Kudo }\end{array}$ & KH5434 & S121 & Soyeop & Leaf & $\begin{array}{l}\text { Atopic } \\
\text { dermatitis }\end{array}$ & Infusion, juice & Oral, topical & 7.69 \\
\hline & & & & & Cattle disease & Infusion & Oral & 25.00 \\
\hline & & & & & Common cold & Decoction, infusion & Oral & 57.69 \\
\hline & & & & & Headache & Decoction & Oral & 1.92 \\
\hline & & & & & Pruritus & Infusion, juice & Oral, topical & 7.69 \\
\hline $\begin{array}{l}\text { Perilla frutescens var. } \\
\text { japonica (Hassk.) H.Hara }\end{array}$ & KH5435 & S122 & Deulggae & Seed & $\begin{array}{l}\text { Abdominal } \\
\text { pain }\end{array}$ & Raw & Oral & 13.73 \\
\hline & & & & & Convulsion & Decoction, oil & Oral & 31.37 \\
\hline & & & & & $\begin{array}{l}\text { Gastroenteric } \\
\text { disorder }\end{array}$ & Seasoned cooked vegetables & Oral & 7.84 \\
\hline & & & & & $\begin{array}{l}\text { Lacquer } \\
\text { poisoning }\end{array}$ & Maceration, powder, raw & Topical & 47.06 \\
\hline Salvia plebeia R.Br. & KH5436 & S164 & Baeamchajeugi & Leaf & Bone diseases & $\begin{array}{l}\text { A sweet drink made from fermented rice, } \\
\text { dried, infusion, }\end{array}$ & Oral & 7.22 \\
\hline & & & & & $\begin{array}{l}\text { Gastroenteric } \\
\text { disorder }\end{array}$ & Infusion & Oral & 5.15 \\
\hline & & & & & Hypofunction & $\begin{array}{l}\text { A sweet drink made from fermented rice, } \\
\text { dried, infusion, }\end{array}$ & Oral & 10.31 \\
\hline & & & & Root & Leg pain & A sweet drink made from fermented rice & Oral & 19.59 \\
\hline & & & & Stem & Arthritis & Infusion & Oral & 3.09 \\
\hline & & & & Whole part & $\begin{array}{l}\text { Abdominal } \\
\text { pain }\end{array}$ & Infusion & Oral & 7.22 \\
\hline & & & & & Arm pain & A sweet drink made from fermented rice & Oral & 5.15 \\
\hline & & & & & Asthma & $\begin{array}{l}\text { A sweet drink made from fermented rice, } \\
\text { dried, infusion, }\end{array}$ & Oral & 4.12 \\
\hline & & & & & Bone diseases & Infusion & Oral & 7.22 \\
\hline & & & & & Cough & Dried, grain syrup, infusion & Oral & 28.87 \\
\hline & & & & & Empyema & Infusion & Oral & 3.09 \\
\hline & & & & & Hypofunction & $\begin{array}{l}\text { A sweet drink made from fermented rice, } \\
\text { brewing }\end{array}$ & Oral & 10.31 \\
\hline & & & & & Leg pain & $\begin{array}{l}\text { A sweet drink made from fermented rice, } \\
\text { infusion }\end{array}$ & Oral & 19.59 \\
\hline & & & & & $\begin{array}{l}\text { Woman } \\
\text { diseases }\end{array}$ & Infusion & Oral & 6.19 \\
\hline
\end{tabular}


Table 2 Plant species for medicinal purposes in community of Gayasan National Park in Korea (Continued)

\begin{tabular}{|c|c|c|c|c|c|c|c|c|c|}
\hline Lardizabalaceae & $\begin{array}{l}\text { Akebia quinata (Houtt.) } \\
\text { Decne. }\end{array}$ & KH5437 & S7 & Eureumdeonggul & Stem & $\begin{array}{l}\text { Abdominal } \\
\text { pain }\end{array}$ & Infusion & Oral & 4.84 \\
\hline & & & & & & Anorexia & Infusion & Oral & 4.84 \\
\hline & & & & & & Arm pain & A sweet drink made from fermented rice & Oral & 6.45 \\
\hline & & & & & & Bone diseases & $\begin{array}{l}\text { A sweet drink made from fermented rice, } \\
\text { infusion }\end{array}$ & Oral & 46.77 \\
\hline & & & & & & Leg pain & A sweet drink made from fermented rice & Oral & 6.45 \\
\hline & & & & & & Pollakiuria & Infusion & Oral & 20.97 \\
\hline & & & & & & Sterility & Infusion & Oral & 9.68 \\
\hline \multirow[t]{5}{*}{ Lauraceae } & Cinnamomum cassia Presl & KH5438 & S44 & Gyepinamu & Bark & Common cold & Decoction & Oral & 100.00 \\
\hline & Lindera obtusiloba Blume & KH5439 & S101 & Saenggangnamu & Stem & Arm pain & A sweet drink made from fermented rice & Oral & 28.00 \\
\hline & & & & & & Leg pain & Brewing & Oral & 28.00 \\
\hline & & & & & & Lumbago & Infusion & Oral & 28.00 \\
\hline & & & & & & $\begin{array}{l}\text { Postpartum } \\
\text { myofascial } \\
\text { pain } \\
\text { syndrome }\end{array}$ & Infusion & Oral & 16.00 \\
\hline \multirow[t]{13}{*}{ Liliaceae } & Allium fistulosum $\mathrm{L}$. & KH5440 & $\$ 9$ & $\mathrm{~Pa}$ & Root & Common cold & Decoction, infusion & Oral & 65.52 \\
\hline & & & & & & Cough & Infusion & Oral & 12.07 \\
\hline & & & & & & Hyperthermia & Infusion & Oral & 22.41 \\
\hline & $\begin{array}{l}\text { Allium scorodorpasum var. } \\
\text { viviparum Regel }\end{array}$ & KH5441 & S10 & Maneul & Bulb & Lymphnoditis & Maceration & Topical & 100.00 \\
\hline & Allium thunbergii G.Don & KH5442 & S11 & Sanbuchu & Aerial part & $\begin{array}{l}\text { Angina } \\
\text { pectoris }\end{array}$ & Kimchi, raw, seasoned cooked vegetables & Oral & 100.00 \\
\hline & $\begin{array}{l}\text { Allium tuberosum Rottler } \\
\text { ex Spreng. }\end{array}$ & KH5443 & S12 & Buchu & Leaf & Epistaxis & Juice & Oral & 18.18 \\
\hline & & & & & & $\begin{array}{l}\text { Lacquer } \\
\text { poisoning }\end{array}$ & Maceration, raw & Topical & 48.48 \\
\hline & & & & & Leaf & Pruritus & Maceration & Topical & 12.12 \\
\hline & & & & & Root & Pollakiuria & Infusion, juice & Oral & 21.21 \\
\hline & $\begin{array}{l}\text { Asparagus schoberioides } \\
\text { Kunth }\end{array}$ & KH5444 & S25 & Bijjaru & Whole part & Edema & Infusion & Oral & 13.46 \\
\hline & & & & & & Pollakiuria & $\begin{array}{l}\text { A sweet drink made from fermented rice, } \\
\text { infusion }\end{array}$ & Oral & 73.08 \\
\hline & & & & & & $\begin{array}{l}\text { Sexual } \\
\text { enhancement }\end{array}$ & Infusion & Oral & 13.46 \\
\hline & $\begin{array}{l}\text { Scilla scilloides (Lindl.) } \\
\text { Druce }\end{array}$ & KH5445 & S170 & Mureut & Root & Edema & Simmer & Oral & 100.00 \\
\hline
\end{tabular}


Table 2 Plant species for medicinal purposes in community of Gayasan National Park in Korea (Continued)

\begin{tabular}{|c|c|c|c|c|c|c|c|c|c|}
\hline Loranthaceae & $\begin{array}{l}\text { Viscum album var. } \\
\text { coloratum (Kom.) Ohwi }\end{array}$ & KH5446 & S193 & Gyeousali & Leaf & Edema & A sweet drink made from fermented rice & Oral & 100.00 \\
\hline & & & & & Stem & Edema & A sweet drink made from fermented rice & Oral & 100.00 \\
\hline Magnoliaceae & Magnolia obovata Thunb. & KH5447 & S106 & Ilbonmokryeon & Fruit & Heart disease & Infusion & Oral & 100.00 \\
\hline \multirow[t]{3}{*}{ Malvaceae } & Althaea rosea (L.) Cav. & KH5448 & $\mathrm{S} 13$ & Jeopsikkot & Root & Leukorrhea & Infusion & Oral & 50.00 \\
\hline & & & & & & Sterility & Infusion & Oral & 50.00 \\
\hline & & & & & Whole part & Sterility & Decoction & Oral & 50.00 \\
\hline Meliaceae & Cedrela sinensis Juss. & KH5449 & $\$ 40$ & Chamjuknamu & Flower & Sterility & Simmer & Oral & 100.00 \\
\hline \multirow[t]{6}{*}{ Menispermaceae } & $\begin{array}{l}\text { Cocculus trilobus (Thunb.) } \\
\text { DC. }\end{array}$ & KH5450 & $\$ 49$ & Daengdaengideonggul & Fruit & Cough & Mixed in ripe persimmon, raw & Oral & 35.29 \\
\hline & & & & & Leaf & $\begin{array}{l}\text { Food } \\
\text { poisoning }\end{array}$ & Infusion & Oral & 11.76 \\
\hline & & & & & Root & $\begin{array}{l}\text { Acute } \\
\text { gastroenteritis }\end{array}$ & Infusion, maceration & Oral & 47.06 \\
\hline & & & & & & Indigestion & Juice & Topical & 5.88 \\
\hline & & & & & Stem & $\begin{array}{l}\text { Acute } \\
\text { gastroenteritis }\end{array}$ & Infusion & Oral & 47.06 \\
\hline & & & & & & $\begin{array}{l}\text { Food } \\
\text { poisoning }\end{array}$ & Infusion & Oral & 11.76 \\
\hline \multirow[t]{14}{*}{ Moraceae } & $\begin{array}{l}\text { Broussonetia kazinoki } \\
\text { Siebold }\end{array}$ & KH5451 & S31 & Daknamu & Fruit & Hookworm & Infusion & Oral & 100.00 \\
\hline & $\begin{array}{l}\text { Cudrania tricuspidata } \\
\text { (Carr.) Bureau ex Lavallee }\end{array}$ & KH5452 & S59 & Kkujippongnamu & Bark & Cancer & Infusion & Oral & 25.31 \\
\hline & & & & & & Glycosuria & Infusion & Oral & 33.44 \\
\hline & & & & & & Liver diseases & Infusion & Oral & 6.56 \\
\hline & & & & & Leaf & $\begin{array}{l}\text { Extravasated } \\
\text { blood }\end{array}$ & Dried, maceration & Oral & 1.25 \\
\hline & & & & & Root & Bone diseases & A sweet drink made from fermented rice & Oral & 17.19 \\
\hline & & & & & & Cancer & $\begin{array}{l}\text { A sweet drink made from fermented rice, } \\
\text { boiling, brewing, infusion }\end{array}$ & Oral & 25.31 \\
\hline & & & & & & $\begin{array}{l}\text { Poor } \\
\text { circulation }\end{array}$ & Infusion & Oral & 1.88 \\
\hline & & & & & & Glycosuria & $\begin{array}{l}\text { A sweet drink made from fermented rice, } \\
\text { brewing, decoction, infusion }\end{array}$ & Oral & 33.44 \\
\hline & & & & & & Hypofunction & $\begin{array}{l}\text { A sweet drink made from fermented rice, } \\
\text { infusion }\end{array}$ & Oral & 7.50 \\
\hline & & & & & & Leg pain & Infusion & Oral & 1.25 \\
\hline & & & & & & Lumbago & Infusion & Oral & 3.75 \\
\hline & & & & & & Neuralgia & Infusion & Oral & 1.25 \\
\hline & & & & & & Paralysis & Infusion & Oral & 0.63 \\
\hline
\end{tabular}


Table 2 Plant species for medicinal purposes in community of Gayasan National Park in Korea (Continued)

\begin{tabular}{|c|c|c|c|c|c|c|c|c|c|}
\hline & & & & & Stem & Bone diseases & $\begin{array}{l}\text { A sweet drink made from fermented rice, } \\
\text { brewing, infusion }\end{array}$ & Oral & 17.19 \\
\hline & & & & & & Cancer & $\begin{array}{l}\text { A sweet drink made from fermented rice, } \\
\text { boiling, brewing, infusion }\end{array}$ & Oral & 25.31 \\
\hline & & & & & & $\begin{array}{l}\text { Poor } \\
\text { circulation }\end{array}$ & Infusion & Oral & 1.88 \\
\hline & & & & & & Glycosuria & $\begin{array}{l}\text { A sweet drink made from fermented rice, } \\
\text { brewing, infusion }\end{array}$ & Oral & 33.44 \\
\hline & & & & & & Hypofunction & $\begin{array}{l}\text { A sweet drink made from fermented rice, } \\
\text { infusion }\end{array}$ & Oral & 7.50 \\
\hline & & & & & & Leg pain & Infusion & Oral & 1.25 \\
\hline & & & & & & Liver diseases & $\begin{array}{l}\text { A sweet drink made from fermented rice, } \\
\text { infusion }\end{array}$ & Oral & 6.56 \\
\hline & & & & & & Lumbago & $\begin{array}{l}\text { A sweet drink made from fermented rice, } \\
\text { infusion }\end{array}$ & Oral & 3.75 \\
\hline & & & & & & Paralysis & Infusion & Oral & 0.63 \\
\hline & Morus alba L. & KH5453 & $\mathrm{S} 108$ & Ppongnamu & Stem & Cancer & Dried, infusion & Oral & 33.33 \\
\hline & & & & & & Glycosuria & Infusion & Oral & 66.67 \\
\hline & & & & & Young leaf & Cancer & Dried, infusion & Oral & 33.33 \\
\hline & & & & & & Glycosuria & Dried, infusion, panbroiled, raw, tea & Oral & 66.67 \\
\hline Nymphaeaceae & Nelumbo nucifera Gaertn. & KH5454 & S109 & Yeonggot & Root & Epistaxis & Infusion, juice & Oral & 100.00 \\
\hline Oleaceae & $\begin{array}{l}\text { Forsythia koreana } \\
\text { (Rehder) Nakai }\end{array}$ & KH5455 & $\mathrm{S} 76$ & Gaenari & Stem & Otalgia & Infusion & Topical & 100.00 \\
\hline Orchidaceae & Gastrodia elata Blume & KH5456 & $\mathrm{S} 79$ & Cheonma & Root & $\begin{array}{l}\text { Lack of } \\
\text { energy }\end{array}$ & Brewing & Oral & 100.00 \\
\hline \multirow[t]{2}{*}{ Paeoniaceae } & Paeonia obovata Maxim. & KH5457 & S116 & Sanjakyak & Root & $\begin{array}{l}\text { Irregular } \\
\text { menstruation }\end{array}$ & Infusion & Oral & 37.50 \\
\hline & & & & & & $\begin{array}{l}\text { Lack of } \\
\text { energy }\end{array}$ & Boiling, decoction & Oral, topical & 62.50 \\
\hline \multirow[t]{5}{*}{ Papaveraceae } & $\begin{array}{l}\text { Chelidonium majus var. } \\
\text { asiaticum (H.Hara) Ohwi }\end{array}$ & KH5458 & 543 & Aegittongpul & Aerial part & Bone diseases & $\begin{array}{l}\text { A sweet drink made from fermented rice, } \\
\text { dried, infusion, }\end{array}$ & Oral & 5.06 \\
\hline & & & & & & $\begin{array}{l}\text { Chronic } \\
\text { myofascial } \\
\text { pain }\end{array}$ & Juice & Oral & 68.35 \\
\hline & & & & & & Hypofunction & $\begin{array}{l}\text { A sweet drink made from fermented rice, } \\
\text { dried, infusion, }\end{array}$ & Oral & 5.06 \\
\hline & & & & & Root & $\begin{array}{l}\text { Chronic } \\
\text { myofascial } \\
\text { pain }\end{array}$ & Brewing & Oral & 68.35 \\
\hline & & & & & Stem & & Brewing & Oral & 68.35 \\
\hline
\end{tabular}


Table 2 Plant species for medicinal purposes in community of Gayasan National Park in Korea (Continued)

\begin{tabular}{|c|c|c|c|c|c|c|c|c|c|}
\hline & & & & & & $\begin{array}{l}\text { Chronic } \\
\text { myofascial } \\
\text { pain }\end{array}$ & & & \\
\hline & & & & & Whole part & $\begin{array}{l}\text { Chronic } \\
\text { myofascial } \\
\text { pain }\end{array}$ & $\begin{array}{l}\text { A sweet drink made from fermented rice, } \\
\text { brewing, dissolution, infusion, juice, } \\
\text { maceration }\end{array}$ & Oral, topical & 68.35 \\
\hline & & & & & & Glycosuria & Brewing & Oral & $\overline{5.06}$ \\
\hline & & & & & & $\begin{array}{l}\text { Induced } \\
\text { abortion }\end{array}$ & Juice & Oral & 11.39 \\
\hline & & & & & & Lumbago & Brewing & Oral & 5.06 \\
\hline & Papaver somniferum L. & KH5459 & S117 & Yanggwibi & Fruit & $\begin{array}{l}\text { Abdominal } \\
\text { pain }\end{array}$ & $\begin{array}{l}\text { Dissolution, dried, grain syrup, juice, } \\
\text { maceration, raw }\end{array}$ & Oral & 53.76 \\
\hline & & & & & & Bone diseases & Infusion & Oral & 6.45 \\
\hline & & & & & & Cattle disease & Dried, dissolution, infusion & Oral & 15.05 \\
\hline & & & & & & Diarrhea & Dried, dissolution & Oral & 2.15 \\
\hline & & & & & & Hypofunction & Infusion & Oral & 8.60 \\
\hline & & & & & & Pain & Dried, dissolution & Oral & 3.23 \\
\hline & & & & & & $\begin{array}{l}\text { Sinews and } \\
\text { joint pain }\end{array}$ & Dissolution & Oral & 3.23 \\
\hline & & & & & & Wound & Dried, dissolution, raw & Topical & 3.23 \\
\hline & & & & & Leaf & $\begin{array}{l}\text { Abdominal } \\
\text { pain }\end{array}$ & Raw & Oral & 53.76 \\
\hline & & & & & Stem & $\begin{array}{l}\text { Abdominal } \\
\text { pain }\end{array}$ & Dried, infusion & Oral & 53.76 \\
\hline & & & & & & Bone diseases & Infusion & Oral & 6.45 \\
\hline & & & & & & Cattle disease & Infusion & Oral & 15.05 \\
\hline & & & & & & Hoove & Infusion & Oral & 4.30 \\
\hline & & & & & Whole part & $\begin{array}{l}\text { Abdominal } \\
\text { pain }\end{array}$ & Brewing & Oral & 53.76 \\
\hline \multirow[t]{5}{*}{ Pedaliaceae } & Sesamum indicum L. & KH5460 & S173 & Chamggae & Seed & $\begin{array}{l}\text { Abdominal } \\
\text { cold } \\
\text { hypersentivity }\end{array}$ & Simmer & Oral & 21.05 \\
\hline & & & & & & Burn & Oil & Topical & 21.05 \\
\hline & & & & & & Convulsion & Oil & Oral & 10.53 \\
\hline & & & & & & Hemorrhaging & Raw, rubbing & Topical & 21.05 \\
\hline & & & & & & Otalgia & Oil & Topical & 26.32 \\
\hline \multirow[t]{3}{*}{ Phytolaccaceae } & $\begin{array}{l}\text { Phytolacca esculenta } \\
\text { VanHoutte }\end{array}$ & KH5461 & $\mathrm{S} 128$ & Jarigong & Root & $\begin{array}{l}\text { Abdominal } \\
\text { pain }\end{array}$ & Infusion & Oral & 10.42 \\
\hline & & & & & & Cattle disease & Juice & Oral & 8.33 \\
\hline & & & & & & Constipation & Raw & Topical & 12.50 \\
\hline
\end{tabular}


Table 2 Plant species for medicinal purposes in community of Gayasan National Park in Korea (Continued)

\begin{tabular}{|c|c|c|c|c|c|c|c|c|c|}
\hline & & & & & & Edema & $\begin{array}{l}\text { A sweet drink made from fermented rice, } \\
\text { infusion }\end{array}$ & Oral & 39.58 \\
\hline & & & & & & Indigestion & Infusion & Oral & 4.17 \\
\hline & & & & & & Lumbago & $\begin{array}{l}\text { A sweet drink made from fermented rice, } \\
\text { brewing, infusion }\end{array}$ & Oral & 25.00 \\
\hline \multirow[t]{6}{*}{ Pinaceae } & $\begin{array}{l}\text { Pinus densiflora Siebold \& } \\
\text { Zucc. }\end{array}$ & KH5462 & $\mathrm{S} 130$ & Sonamu & Leaf & Dental pain & Raw & Topical & 33.33 \\
\hline & & & & & & Lumbago & Steamed & Topical & 11.11 \\
\hline & & & & & Pollen & Hemorrhaging & Powder & Topical & 22.22 \\
\hline & & & & & Stem & Otalgia & Infusion & Topical & 33.33 \\
\hline & $\begin{array}{l}\text { Pinus koraiensis Siebold \& } \\
\text { Zucc. }\end{array}$ & KH5463 & $\mathrm{S} 131$ & Jatnamu & Pine resin & Boils & Raw & Topical & 44.44 \\
\hline & & & & & & Wound & Melt & Topical & 55.56 \\
\hline \multirow[t]{8}{*}{ Plantaginaceae } & Plantago asiatica L. & KH5464 & $\mathrm{S} 132$ & Jilgyeongi & Leaf & Heart disease & Infusion & Oral & 4.97 \\
\hline & & & & & Root & $\begin{array}{l}\text { Abdominal } \\
\text { pain }\end{array}$ & Juice & Oral & 0.62 \\
\hline & & & & & & Pollakiuria & Infusion & Oral & 78.26 \\
\hline & & & & & Whole part & Epistaxis & Dried,infusion, raw & Oral & 8.70 \\
\hline & & & & & & $\begin{array}{l}\text { Gastroenteric } \\
\text { disorder }\end{array}$ & Decoction & Oral & 3.73 \\
\hline & & & & & & Heart disease & Infusion & Oral & 4.97 \\
\hline & & & & & & Pollakiuria & Decoction, dried, infusion, raw & Oral & 78.26 \\
\hline & & & & & & Sterility & Infusion & Oral & 3.73 \\
\hline \multirow[t]{10}{*}{ Poaceae } & $\begin{array}{l}\text { Hordeum vulgare var. } \\
\text { hexastichon (L.) Asch. }\end{array}$ & KH5465 & S87 & Bori & Malt & $\begin{array}{l}\text { Abdominal } \\
\text { pain }\end{array}$ & Malted barley seedling & Oral & 0.62 \\
\hline & & & & & & Arm pain & $\begin{array}{l}\text { A sweet drink made from fermented rice, } \\
\text { infusion }\end{array}$ & Oral & 6.69 \\
\hline & & & & & & Arthritis & $\begin{array}{l}\text { A sweet drink made from fermented rice, } \\
\text { infusion }\end{array}$ & Oral & 1.87 \\
\hline & & & & & & Bone diseases & $\begin{array}{l}\text { A sweet drink made from fermented rice, } \\
\text { brewing, dried, infusion }\end{array}$ & Oral & 29.70 \\
\hline & & & & & & Cancer & A sweet drink made from fermented rice & Oral & 0.47 \\
\hline & & & & & & $\begin{array}{l}\text { Carpal tunnel } \\
\text { syndrome }\end{array}$ & A sweet drink made from fermented rice & Oral & 1.09 \\
\hline & & & & & & $\begin{array}{l}\text { Chronic } \\
\text { myofascial } \\
\text { pain }\end{array}$ & A sweet drink made from fermented rice & Oral & 1.09 \\
\hline & & & & & & Common cold & Decoction & Oral & 0.78 \\
\hline & & & & & & Cough & A sweet drink made from fermented rice & Oral & 1.87 \\
\hline & & & & & & Edema & A sweet drink made from fermented rice & Oral & 1.87 \\
\hline
\end{tabular}


Table 2 Plant species for medicinal purposes in community of Gayasan National Park in Korea (Continued)

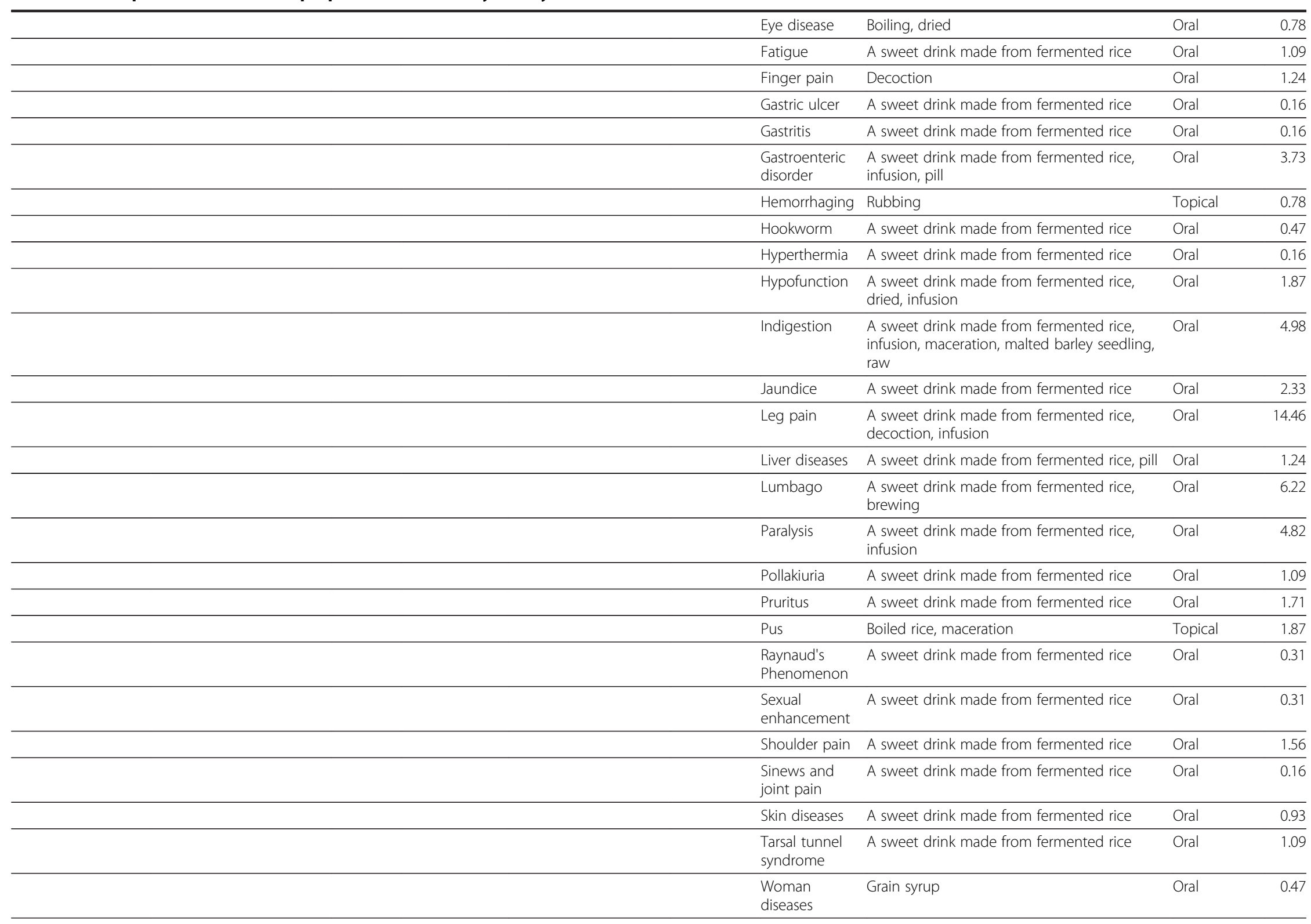


Table 2 Plant species for medicinal purposes in community of Gayasan National Park in Korea (Continued)

\begin{tabular}{|c|c|c|c|c|c|c|c|c|}
\hline $\begin{array}{l}\text { Hordeum vulgare var. } \\
\text { nudum Spenn. }\end{array}$ & KH5466 & 588 & Ssalbori & Seed & Pollakiuria & A sweet drink made from fermented rice & Oral & 100.00 \\
\hline $\begin{array}{l}\text { Imperata cylindrica var. } \\
\text { koenigii (Retz.) Pilg. }\end{array}$ & KH5467 & 592 & Tti & Flower & $\begin{array}{l}\text { Bloody } \\
\text { discharge }\end{array}$ & Infusion & Oral & 15.38 \\
\hline & & & & & Burn & Burn, raw & Topical & 84.62 \\
\hline Oryza sativa $\mathrm{L}$. & KH5468 & S113 & Byeo & Root & $\begin{array}{l}\text { Lacquer } \\
\text { poisoning }\end{array}$ & Infusion & Topical & 48.98 \\
\hline & & & & Seed & $\begin{array}{l}\text { Carpal tunnel } \\
\text { syndrome }\end{array}$ & Infusion & Oral & 4.08 \\
\hline & & & & & Hangover & Powder & Oral & 4.08 \\
\hline & & & & & Jaundice & A sweet drink made from fermented rice & Oral & 5.10 \\
\hline & & & & & $\begin{array}{l}\text { Lacquer } \\
\text { poisoning }\end{array}$ & Maceration, raw & Topical & 48.98 \\
\hline & & & & & Pollakiuria & Infusion, rice water & Oral & 9.18 \\
\hline & & & & & Snakebite & Porridge, raw & Topical & 8.16 \\
\hline & & & & & $\begin{array}{l}\text { Tarsal tunnel } \\
\text { syndrome }\end{array}$ & Infusion & Oral & 4.08 \\
\hline & & & & Stem & Burn & Raw & Topical & 2.04 \\
\hline & & & & & Hemorrhaging & Raw & Topical & 2.04 \\
\hline & & & & & Indigestion & Dried, infusion & Oral & 2.04 \\
\hline & & & & & Urticaria & Roast & Topical & 5.10 \\
\hline & & & & & Wound & Raw & Topical & 5.10 \\
\hline $\begin{array}{l}\text { Oryza sativa var. glutinosa } \\
\text { Blanco }\end{array}$ & KH5469 & S114 & Chalbyeo & Seed & $\begin{array}{l}\text { Abdominal } \\
\text { cold } \\
\text { hypersentivity }\end{array}$ & Pill, simmer & Oral & 31.25 \\
\hline & & & & & Anorexia & Pill, simmer & Oral & 7.81 \\
\hline & & & & & $\begin{array}{l}\text { Carpal tunnel } \\
\text { syndrome }\end{array}$ & A sweet drink made from fermented rice & Oral & 3.13 \\
\hline & & & & & $\begin{array}{l}\text { Lack of } \\
\text { energy }\end{array}$ & Pill, simmer & Oral & 7.81 \\
\hline & & & & & Paralysis & A sweet drink made from fermented rice & Oral & 12.50 \\
\hline & & & & & Pollakiuria & Porridge & Oral & 7.81 \\
\hline & & & & & Sterility & Pill, simmer & Oral & 7.81 \\
\hline & & & & & $\begin{array}{l}\text { Tarsal tunnel } \\
\text { syndrome }\end{array}$ & A sweet drink made from fermented rice & Oral & 3.13 \\
\hline & & & & Stem & Cattle disease & Fumigation & Topical & 7.81 \\
\hline & & & & & Urticaria & Fumigation & Topical & 7.81 \\
\hline & & & & Young leaf & Epistaxis & Infusion & Oral & 3.13 \\
\hline
\end{tabular}


Table 2 Plant species for medicinal purposes in community of Gayasan National Park in Korea (Continued)

\begin{tabular}{|c|c|c|c|c|c|c|c|c|}
\hline $\begin{array}{l}\text { Phyllostachys bambusoides } \\
\text { Siebold \& Zucc. }\end{array}$ & KH5470 & S126 & Wangdae & Leaf & Common cold & Infusion & Oral & 38.46 \\
\hline & & & & Root & Lumbago & A sweet drink made from fermented rice & Oral & 61.54 \\
\hline $\begin{array}{l}\text { Sasa borealis (Hack.) } \\
\text { Makino }\end{array}$ & KH5471 & S167 & Joritdae & Aerial part & Cattle disease & Infusion & Oral & 100.00 \\
\hline Sasa coreana Nakai & KH5472 & $\mathrm{S} 168$ & Sinidae & Leaf & Common cold & Decoction, infusion & Oral & 56.25 \\
\hline & & & & Stem & Cattle disease & Infusion & Oral & 43.75 \\
\hline Setaria italica (L.) P.Beauv. & KH5473 & S174 & Jo & Aerial part & Cattle disease & Infusion & Oral & 100.00 \\
\hline Triticum aestivum L. & KH5474 & S187 & Mil & Seed & Arm pain & $\begin{array}{l}\text { A sweet drink made from fermented rice, } \\
\text { brewing }\end{array}$ & Oral & 5.26 \\
\hline & & & & & Asthma & Brewing & Oral & 0.58 \\
\hline & & & & & Boils & Dough & Topical & 2.05 \\
\hline & & & & & Bone diseases & $\begin{array}{l}\text { A sweet drink made from fermented rice, } \\
\text { brewing, dough, infusion }\end{array}$ & Oral, topical & 19.30 \\
\hline & & & & & Bruising & Dough & Topical & 9.94 \\
\hline & & & & & $\begin{array}{l}\text { Carpal tunnel } \\
\text { syndrome }\end{array}$ & $\begin{array}{l}\text { A sweet drink made from fermented rice, } \\
\text { brewing, dough }\end{array}$ & Oral, topical & 2.05 \\
\hline & & & & & Cattle disease & Brewing & Oral & 0.88 \\
\hline & & & & & $\begin{array}{l}\text { Chronic } \\
\text { myofascial } \\
\text { pain }\end{array}$ & $\begin{array}{l}\text { Brewing, chopped noodles, clear soup with } \\
\text { flour dumplings, dissolution, dried, mixed in } \\
\text { liquor, powder, roast }\end{array}$ & Oral & 9.94 \\
\hline & & & & & Common cold & Brewing, tea & Oral & 2.92 \\
\hline & & & & & Cough & Brewing & Oral & 1.46 \\
\hline & & & & & $\begin{array}{l}\text { Extravasated } \\
\text { blood }\end{array}$ & Dough & Topical & 0.29 \\
\hline & & & & & $\begin{array}{l}\text { Gastroenteric } \\
\text { disorder }\end{array}$ & $\begin{array}{l}\text { A sweet drink made from fermented rice, } \\
\text { brewing, juice }\end{array}$ & Oral & 5.56 \\
\hline & & & & & Hangover & Clear soup with flour dumplings & Oral & 1.75 \\
\hline & & & & & Hookworm & Infusion & Oral & 2.05 \\
\hline & & & & & Hyperthermia & Brewing & Oral & 0.29 \\
\hline & & & & & Indigestion & Clear soup with flour dumplings, extraction & Oral & 2.92 \\
\hline & & & & & Leg pain & $\begin{array}{l}\text { A sweet drink made from fermented rice, } \\
\text { brewing }\end{array}$ & Oral & 12.28 \\
\hline & & & & & Lumbago & Brewing, powder & Oral & 8.48 \\
\hline & & & & & Milk fever & Clear soup with flour dumplings & Oral & 2.05 \\
\hline & & & & & Orchiopathy & Brewing & Oral & 0.58 \\
\hline & & & & & Paralysis & Brewing & Oral & 1.17 \\
\hline & & & & & Pus & Dough, powder & Topical & 2.92 \\
\hline & & & & & Sprain & Dough & Topical & 2.63 \\
\hline
\end{tabular}


Table 2 Plant species for medicinal purposes in community of Gayasan National Park in Korea (Continued)

\begin{tabular}{|c|c|c|c|c|c|c|c|c|c|}
\hline & & & & & & $\begin{array}{l}\text { Tarsal tunnel } \\
\text { syndrome }\end{array}$ & $\begin{array}{l}\text { A sweet drink made from fermented rice, } \\
\text { brewing, dough }\end{array}$ & Oral, topical & 2.63 \\
\hline & Zea mays $\mathrm{L}$. & KH5475 & S198 & Oksusu & Fruit & Pollakiuria & Tea & Oral & 94.62 \\
\hline & & & & & Style & $\begin{array}{l}\text { Abdominal } \\
\text { pain }\end{array}$ & Infusion & Oral & 2.31 \\
\hline & & & & & & Anorexia & Infusion & Oral & 2.31 \\
\hline & & & & & & Lung diseases & Infusion & Oral & 0.77 \\
\hline & & & & & & Pollakiuria & Decoction, dried, infusion & Oral & 94.62 \\
\hline \multirow[t]{13}{*}{ Polygonaceae } & $\begin{array}{l}\text { Fagopyrum esculentum } \\
\text { Moench }\end{array}$ & KH5476 & S72 & Memil & Pericarp & Pus & Maceration & Topical & 100.00 \\
\hline & $\begin{array}{l}\text { Fallopia japonica (Houtt.) } \\
\text { RonseDecr. }\end{array}$ & KH5477 & $\mathrm{S} 73$ & Hojanggeun & Aerial part & Pollakiuria & Infusion & Oral & 100.00 \\
\hline & & & & & Root & Pollakiuria & $\begin{array}{l}\text { A sweet drink made from fermented rice, } \\
\text { infusion }\end{array}$ & Oral & 100.00 \\
\hline & & & & & Whole part & Pollakiuria & Infusion & Oral & 100.00 \\
\hline & $\begin{array}{l}\text { Fallopia multiflora } \\
\text { (Thunb.) Haraldson }\end{array}$ & KH5478 & S74 & Hasuo & Root & $\begin{array}{l}\text { Lack of } \\
\text { energy }\end{array}$ & Dried, powder & Oral & 9.09 \\
\hline & & & & & & Sthenia & Brewing & Oral, topical & 90.91 \\
\hline & Rumex acetosa $\mathrm{L}$. & KH5479 & $S 160$ & Suyeong & Whole part & Pollakiuria & Infusion & Oral & 41.18 \\
\hline & & & & & & $\begin{array}{l}\text { Prostate } \\
\text { disease }\end{array}$ & Infusion & Oral & 41.18 \\
\hline & & & & & & Renal disease & Infusion & Oral & 17.65 \\
\hline & Rumex crispus L. & KH5480 & S161 & Sorijaengi & Root & Leg pain & A sweet drink made from fermented rice & Oral & 38.89 \\
\hline & & & & & & Malaria & Maceration & Oral & 27.78 \\
\hline & & & & & Whole part & Leg pain & Brewing & Oral & 38.89 \\
\hline & & & & & & Lumbago & Brewing & Oral & 33.33 \\
\hline \multirow[t]{2}{*}{ Polyporaceae } & $\begin{array}{l}\text { Coriolus versicolor (L.: Fr.) } \\
\text { Quél. }\end{array}$ & KH5481 & S54 & Gureumbeoseot & Whole part & Common cold & Boiling, decoction & Oral & 100.00 \\
\hline & Poria cocos Wolf & KH5482 & S137 & Bokryeong & Whole part & Hoove & Powder & Oral & 100.00 \\
\hline \multirow[t]{2}{*}{ Portulacaceae } & Portulaca oleracea $\mathrm{L}$. & KH5483 & S138 & Soebireum & Aerial part & Cancer & Infusion & Oral & 83.33 \\
\hline & & & & & & Diarrhea & Seasoned cooked vegetables & Oral & 16.67 \\
\hline \multirow[t]{5}{*}{ Punicaceae } & Punica granatum L. & KH5484 & S145 & Seokryunamu & Flower & Epistaxis & Dried, infusion & Topical & 20.69 \\
\hline & & & & & Fruit & $\begin{array}{l}\text { Abdominal } \\
\text { pain }\end{array}$ & Raw & Oral & 13.79 \\
\hline & & & & & & Common cold & Extraction, infusion, raw & Oral & 41.38 \\
\hline & & & & & & Cough & Infusion & Oral & 24.14 \\
\hline & & & & & Pericarp & Epistaxis & Dried & Topical & 20.69 \\
\hline
\end{tabular}


Table 2 Plant species for medicinal purposes in community of Gayasan National Park in Korea (Continued)

\begin{tabular}{|c|c|c|c|c|c|c|c|c|c|}
\hline Ramariaceae & $\begin{array}{l}\text { Ramaria botrytis (Pers.) } \\
\text { Ricken }\end{array}$ & KH5485 & S149 & Ssaribeoseot & Whole part & Diarrhea & Infusion & Oral & 100.00 \\
\hline \multirow[t]{21}{*}{ Ranunculaceae } & Aconitum ciliare DC. & KH5486 & S3 & Notjeotgaraknamul & Root & Afterpain & Infusion & Oral & 1.08 \\
\hline & & & & & & Arm pain & $\begin{array}{l}\text { A sweet drink made from fermented rice, } \\
\text { infusion }\end{array}$ & Oral & 8.92 \\
\hline & & & & & & Bone diseases & $\begin{array}{l}\text { A sweet drink made from fermented rice, } \\
\text { infusion }\end{array}$ & Oral & 6.22 \\
\hline & & & & & & $\begin{array}{l}\text { Carpal tunnel } \\
\text { syndrome }\end{array}$ & $\begin{array}{l}\text { A sweet drink made from fermented rice, } \\
\text { decoction, infusion, simmer }\end{array}$ & Oral & 15.41 \\
\hline & & & & & & Leg pain & $\begin{array}{l}\text { A sweet drink made from fermented rice, } \\
\text { decoction, infusion }\end{array}$ & Oral & 14.05 \\
\hline & & & & & & Lumbago & Infusion & Oral & 0.81 \\
\hline & & & & & & Malgia & Infusion & Oral & 0.27 \\
\hline & & & & & & Paralysis & $\begin{array}{l}\text { A sweet drink made from fermented rice, } \\
\text { brewing, decoction, infusion, simmer }\end{array}$ & Oral & 37.84 \\
\hline & & & & & & $\begin{array}{l}\text { Postpartum } \\
\text { myofascial } \\
\text { pain } \\
\text { syndrome }\end{array}$ & Infusion & Oral & 1.08 \\
\hline & & & & & & $\begin{array}{l}\text { Tarsal tunnel } \\
\text { syndrome }\end{array}$ & $\begin{array}{l}\text { A sweet drink made from fermented rice, } \\
\text { decoction, infusion, simmer }\end{array}$ & Oral & 14.32 \\
\hline & $\begin{array}{l}\text { Aconitum coreanum (H. } \\
\text { Lév.) Rapaics }\end{array}$ & KH5487 & S4 & Baekbuja & Stem & Bone diseases & Infusion & Oral & 50.00 \\
\hline & & & & & & $\begin{array}{l}\text { Sinews and } \\
\text { joint pain }\end{array}$ & Infusion & Oral & 50.00 \\
\hline & $\begin{array}{l}\text { Pulsatilla koreana (Yabe } \\
\text { ex Nakai) Nakai ex Nakai }\end{array}$ & KH5488 & S144 & Halmikkot & Leaf & $\begin{array}{l}\text { Facial nerve } \\
\text { paralysis }\end{array}$ & Maceration & Topical & 2.99 \\
\hline & & & & & Root & $\begin{array}{l}\text { Abdominal } \\
\text { pain }\end{array}$ & $\begin{array}{l}\text { A sweet drink made from fermented rice, } \\
\text { grain syrup, infusion }\end{array}$ & Oral & 11.19 \\
\hline & & & & & & Bone diseases & $\begin{array}{l}\text { A sweet drink made from fermented rice, } \\
\text { brewing }\end{array}$ & Oral & 2.99 \\
\hline & & & & & & Convulsion & Infusion & Oral & 2.99 \\
\hline & & & & & & $\begin{array}{l}\text { Facial nerve } \\
\text { paralysis }\end{array}$ & Maceration & Topical & 2.99 \\
\hline & & & & & & $\begin{array}{l}\text { Gastroenteric } \\
\text { disorder }\end{array}$ & $\begin{array}{l}\text { A sweet drink made from fermented rice, } \\
\text { infusion, simmer }\end{array}$ & Oral & 8.96 \\
\hline & & & & & & Hookworm & $\begin{array}{l}\text { A sweet drink made from fermented rice, } \\
\text { decoction, dissolution, grain syrup, infusion, } \\
\text { juice, maceration }\end{array}$ & Oral, topical & 60.45 \\
\hline & & & & & & Hypoacusis & Brewing & Oral & 3.73 \\
\hline & & & & & & Lumbago & $\begin{array}{l}\text { A sweet drink made from fermented rice, } \\
\text { brewing }\end{array}$ & Oral & 2.99 \\
\hline
\end{tabular}


Table 2 Plant species for medicinal purposes in community of Gayasan National Park in Korea (Continued)

\begin{tabular}{|c|c|c|c|c|c|c|c|c|c|}
\hline & & & & & & Lymphnoditis & Maceration & Topical & 3.73 \\
\hline & Ranunculus sceleratus L. & KH5489 & S150 & Gaegurijari & Leaf & Callus & Maceration & Topical & 12.07 \\
\hline & & & & & & $\begin{array}{l}\text { Facial nerve } \\
\text { paralysis }\end{array}$ & Maceration, raw & Topical & 87.93 \\
\hline & & & & & Root & $\begin{array}{l}\text { Facial nerve } \\
\text { paralysis }\end{array}$ & Maceration & Topical & 87.93 \\
\hline \multirow[t]{20}{*}{ Rhamnaceae } & Hovenia dulcis Thunb. & KH5490 & S89 & Heotgaenamu & Fruit & Cancer & Decoction & Oral & 5.56 \\
\hline & & & & & & Liver diseases & Tea & Oral & 69.44 \\
\hline & & & & & Stem & Cancer & Decoction & Oral & 5.56 \\
\hline & & & & & & Glycosuria & Infusion & Oral & 13.89 \\
\hline & & & & & & Hangover & Brewing, infusion & Oral & 11.11 \\
\hline & & & & & & Liver diseases & Infusion & Oral & 69.44 \\
\hline & $\begin{array}{l}\text { Zizyphus jujuba var. } \\
\text { inermis (Bunge) Rehder }\end{array}$ & KH5491 & S200 & Daechunamu & Fruit & $\begin{array}{l}\text { Abdominal } \\
\text { cold } \\
\text { hypersentivity }\end{array}$ & Decoction, grain syrup, infusion, pill, simmer & Oral & 14.29 \\
\hline & & & & & & Anorexia & Pill, simmer & Oral & 2.38 \\
\hline & & & & & & Common cold & Boiling, decoction, dried, roast & Oral & 2.38 \\
\hline & & & & & & Cough & Infusion & Oral & 8.10 \\
\hline & & & & & & Fatigue & $\begin{array}{l}\text { A sweet drink made from fermented rice, } \\
\text { boiled rice, raw }\end{array}$ & Oral & 10.00 \\
\hline & & & & & & $\begin{array}{l}\text { Gastroenteric } \\
\text { disorder }\end{array}$ & Infusion & Oral & 0.95 \\
\hline & & & & & & $\begin{array}{l}\text { Lack of } \\
\text { energy }\end{array}$ & Decoction, pill, simmer, tea & Oral & 3.33 \\
\hline & & & & & & Sterility & Grain syrup, infusion, pill, simmer & Oral & 8.10 \\
\hline & & & & & & $\begin{array}{l}\text { Woman } \\
\text { diseases }\end{array}$ & Infusion & Oral & 3.81 \\
\hline & & & & & Leaf & Overheating & Raw & Topical & 0.48 \\
\hline & & & & & Root & Sterility & Infusion & Oral & 8.10 \\
\hline & & & & & Stem & Arm pain & Infusion & Oral & 0.95 \\
\hline & & & & & & $\begin{array}{l}\text { Facial nerve } \\
\text { paralysis }\end{array}$ & Raw & Topical & 44.29 \\
\hline & & & & & & Leg pain & Infusion & Oral & 0.95 \\
\hline \multirow[t]{3}{*}{ Rosaceae } & $\begin{array}{l}\text { Chaenomeles sinensis } \\
\text { (Thouin) Koehne }\end{array}$ & KH5492 & 542 & Mogwanamu & Fruit & Common cold & $\begin{array}{l}\text { Boiling, brewing, decoction, extraction, } \\
\text { infusion }\end{array}$ & Oral & 45.36 \\
\hline & & & & & & Cough & $\begin{array}{l}\text { Boiling, brewing, decoction, dissolution, } \\
\text { extraction, infusion, steamed }\end{array}$ & Oral & 54.64 \\
\hline & $\begin{array}{l}\text { Duchesnea indica (Andr.) } \\
\text { Focke }\end{array}$ & KH5493 & $\mathrm{S} 64$ & Baemddalgi & Fruit & Dental pain & Infusion & Oral & 46.15 \\
\hline
\end{tabular}


Table 2 Plant species for medicinal purposes in community of Gayasan National Park in Korea (Continued)

\begin{tabular}{|c|c|c|c|c|c|c|c|c|}
\hline & & & & & Snakebite & Maceration & Topical & $\overline{38.46}$ \\
\hline & & & & Whole part & Dental pain & Infusion & Topical & 46.15 \\
\hline & & & & & Measles & Infusion & Oral & 15.38 \\
\hline Potentilla chinensis Ser. & KH5494 & S139 & Ttakjikkoch & Root & $\begin{array}{l}\text { Gastroenteric } \\
\text { disorder }\end{array}$ & Infusion, raw & Oral & 100.00 \\
\hline $\begin{array}{l}\text { Prunus armeniaca var. } \\
\text { ansu Maxim. }\end{array}$ & KH5495 & S140 & Salgunamu & Seed & Indigestion & Maceration & Oral & 100.00 \\
\hline $\begin{array}{l}\text { Prunus davidiana } \\
\text { (Carrière) Franch. }\end{array}$ & KH5496 & S141 & Sanboksanamu & Fruit & $\begin{array}{l}\text { Abdominal } \\
\text { pain }\end{array}$ & Extraction & Oral & 6.15 \\
\hline & & & & & Arm pain & Brewing, extraction & Oral & 21.54 \\
\hline & & & & & Hypertension & Brewing & Oral & 4.62 \\
\hline & & & & & Leg pain & Brewing, extraction & Oral & 44.62 \\
\hline & & & & & Lumbago & Brewing & Oral & 9.23 \\
\hline & & & & & Osteoporosis & Extraction & Oral & 1.54 \\
\hline & & & & Root & Leg pain & Brewing & Oral & 44.62 \\
\hline & & & & & Lumbago & Brewing & Oral & 9.23 \\
\hline & & & & Stem & Asthma & Grain syrup & Oral & $\overline{4.62}$ \\
\hline & & & & & Bone diseases & Infusion & Oral & 7.69 \\
\hline & & & & & Leg pain & Infusion & Oral & 44.62 \\
\hline $\begin{array}{l}\text { Prunus mume Siebold \& } \\
\text { Zucc. }\end{array}$ & KH5497 & S142 & Maesilnamu & Fruit & $\begin{array}{l}\text { Abdominal } \\
\text { pain }\end{array}$ & Extraction & Oral & 46.00 \\
\hline & & & & & $\begin{array}{l}\text { Gastroenteric } \\
\text { disorder }\end{array}$ & Extraction & Oral & 10.00 \\
\hline & & & & & Indigestion & Brewing, extraction & Oral & 44.00 \\
\hline $\begin{array}{l}\text { Pyrus pyrifolia (Burm.f.) } \\
\text { Nakai }\end{array}$ & KH5498 & S146 & Dolbaenamu & Fruit & Asthma & Fermentation & Oral & 40.00 \\
\hline & & & & & Cough & Fermentation & Oral & 40.00 \\
\hline & & & & & Leg pain & Brewing, raw & Oral & 20.00 \\
\hline $\begin{array}{l}\text { Pyrus pyrifolia var. culta } \\
\text { (Makino) Nakai }\end{array}$ & KH5499 & S147 & Baenamu & Fruit & Bronchitis & Extraction, infusion & Oral & 5.88 \\
\hline & & & & & Common cold & $\begin{array}{l}\text { A sweet drink made from fermented rice, } \\
\text { decoction, fermentation, infusion, raw, roast }\end{array}$ & Oral & 56.86 \\
\hline & & & & & Cough & Infusion, raw, roast, steamed & Oral & 33.33 \\
\hline & & & & & Indigestion & Infusion & Oral & 3.92 \\
\hline Rosa multiflora Thunb. & KH5500 & S158 & Jjilrekkot & Root & Osteoporosis & Infusion & Oral & 100.00 \\
\hline Rubus coreanus Miq. & KH5501 & S159 & Bokbunjaddalgi & Fruit & $\begin{array}{l}\text { Sexual } \\
\text { enhancement }\end{array}$ & Extraction, raw & Oral & 100.00 \\
\hline Sanguisorba officinalis L. & KH5502 & S165 & Oipul & Leaf & Cattle disease & Juice & Oral & 7.69 \\
\hline
\end{tabular}


Table 2 Plant species for medicinal purposes in community of Gayasan National Park in Korea (Continued)

\begin{tabular}{|c|c|c|c|c|c|c|c|c|c|}
\hline & & & & & & Hoove & Juice & Oral & 7.69 \\
\hline & & & & & Root & Cattle disease & Juice & Oral & 7.69 \\
\hline & & & & & & Finger pain & Infusion & Topical & 3.85 \\
\hline & & & & & & Hoove & Juice & Oral & 7.69 \\
\hline & & & & & & Hypofunction & Decoction, simmer & Oral, topical & 7.69 \\
\hline & & & & & & Leg pain & Infusion & Topical & 23.08 \\
\hline & & & & & & Pus & Infusion & Topical & 3.85 \\
\hline & & & & & & Sprain & Infusion & Topical & 19.23 \\
\hline & & & & & & Toe pain & Infusion & Topical & 3.85 \\
\hline & & & & & Whole part & Dysentery & Infusion & Oral & 23.08 \\
\hline & Sorbus commixta Hedl. & KH5503 & S181 & Magamok & Stem & Arm pain & A sweet drink made from fermented rice & Oral & 25.00 \\
\hline & & & & & & Leg pain & A sweet drink made from fermented rice & Oral & 25.00 \\
\hline & & & & & & Shoulder pain & A sweet drink made from fermented rice & Oral & 50.00 \\
\hline & $\begin{array}{l}\text { Spiraea prunifolia f. } \\
\text { simpliciflora Nakai }\end{array}$ & KH5504 & S182 & Jopapnamu & Root & Indigestion & Infusion & Oral & 94.12 \\
\hline & & & & & Stem & Indigestion & Infusion & Oral & 94.12 \\
\hline & & & & & & Leg pain & Infusion & Oral & 5.88 \\
\hline \multirow[t]{8}{*}{ Rubiaceae } & $\begin{array}{l}\text { Gardenia jasminoides J. } \\
\text { Ellis }\end{array}$ & KH5505 & 578 & Chijanamu & Fruit & Bone diseases & Dough & Topical & 1.43 \\
\hline & & & & & & Bruising & Dough, infusion & Topical & 61.43 \\
\hline & & & & & & $\begin{array}{l}\text { Carpal tunnel } \\
\text { syndrome }\end{array}$ & Infusion & Topical & 2.86 \\
\hline & & & & & & $\begin{array}{l}\text { Chronic } \\
\text { myofascial } \\
\text { pain }\end{array}$ & Infusion & Topical & 4.29 \\
\hline & & & & & & $\begin{array}{l}\text { Extravasated } \\
\text { blood }\end{array}$ & Dough & Topical & 1.43 \\
\hline & & & & & & Pus & Brewing & Oral & 5.71 \\
\hline & & & & & & Sprain & Dough, infusion & Topical & 20.00 \\
\hline & & & & & & $\begin{array}{l}\text { Tarsal tunnel } \\
\text { syndrome }\end{array}$ & Infusion & Topical & 2.86 \\
\hline \multirow[t]{6}{*}{ Rutaceae } & Citrus unshiu S.Marcov. & KH5506 & 547 & Gyul & Pericarp & Common cold & Infusion & Oral & 100.00 \\
\hline & $\begin{array}{l}\text { Phellodendron amurense } \\
\text { Rupr. }\end{array}$ & KH5507 & S125 & Hwangbyeoknamu & Bark & Arm pain & A sweet drink made from fermented rice & Oral & 4.12 \\
\hline & & & & & & Bone diseases & Brewing & Oral & 61.42 \\
\hline & & & & & & Hyperthermia & Brewing & Oral & 0.37 \\
\hline & & & & & & Indigestion & Infusion & Oral & 3.75 \\
\hline & & & & & & Leg pain & & Oral & 18.35 \\
\hline
\end{tabular}


Table 2 Plant species for medicinal purposes in community of Gayasan National Park in Korea (Continued)

\begin{tabular}{|c|c|c|c|c|c|c|c|c|}
\hline & & & & & & \multicolumn{3}{|l|}{$\begin{array}{l}\text { A sweet drink made from fermented rice, } \\
\text { infusion }\end{array}$} \\
\hline & & & & & Lumbago & $\begin{array}{l}\text { A sweet drink made from fermented rice, } \\
\text { infusion }\end{array}$ & Oral & 11.24 \\
\hline & & & & Endodermis & Indigestion & Steep & Oral & 3.75 \\
\hline & & & & & Lumbago & Brewing & Oral & 11.24 \\
\hline & & & & Stem & Arm pain & A sweet drink made from fermented rice & Oral & 4.12 \\
\hline & & & & & Bone diseases & $\begin{array}{l}\text { A sweet drink made from fermented rice, } \\
\text { brewing, infusion }\end{array}$ & Oral & 61.42 \\
\hline & & & & & Leg pain & $\begin{array}{l}\text { A sweet drink made from fermented rice, } \\
\text { infusion }\end{array}$ & Oral & 18.35 \\
\hline & & & & & Lumbago & $\begin{array}{l}\text { A sweet drink made from fermented rice, } \\
\text { infusion }\end{array}$ & Oral & 11.24 \\
\hline & & & & & Neuralgia & Infusion & Oral & 0.75 \\
\hline Poncirus trifoliata (L.) Raf. & KH5508 & S135 & Taengjanamu & Fruit & Cough & Dried, infusion & Oral & 14.00 \\
\hline & & & & & $\begin{array}{l}\text { Lacquer } \\
\text { poisoning }\end{array}$ & Infusion & Topical & 12.00 \\
\hline & & & & & Urticaria & Infusion & Oral, topical & 66.00 \\
\hline & & & & Leaf & Snakebite & Maceration & Topical & 8.00 \\
\hline $\begin{array}{l}\text { Zanthoxylum piperitum } \\
\text { (L.) DC. }\end{array}$ & KH5509 & S196 & Chopinamu & Fruit & Asthma & Oil & Oral & 0.32 \\
\hline & & & & & $\begin{array}{l}\text { Carpal tunnel } \\
\text { syndrome }\end{array}$ & Brewing, infusion & Oral & 4.05 \\
\hline & & & & & Dental pain & Infusion, raw & Topical & 1.78 \\
\hline & & & & & $\begin{array}{l}\text { Gastroenteric } \\
\text { disorder }\end{array}$ & Oil & Oral & 0.32 \\
\hline & & & & & Leg pain & Brewing & Oral & 14.08 \\
\hline & & & & & Paralysis & $\begin{array}{l}\text { A sweet drink made from fermented rice, } \\
\text { infusion, oil, simmer }\end{array}$ & Oral & 25.24 \\
\hline & & & & & $\begin{array}{l}\text { Tarsal tunnel } \\
\text { syndrome }\end{array}$ & Brewing, infusion & Oral & 4.05 \\
\hline & & & & Pericarp & $\begin{array}{l}\text { Carpal tunnel } \\
\text { syndrome }\end{array}$ & Infusion & Oral & 4.05 \\
\hline & & & & & Eye disease & Infusion & Oral & 0.32 \\
\hline & & & & & $\begin{array}{l}\text { Facial nerve } \\
\text { paralysis }\end{array}$ & Infusion & Oral & 1.62 \\
\hline & & & & & Headache & Infusion & Oral & 0.65 \\
\hline & & & & & Hypertension & Powder & Oral & 1.29 \\
\hline & & & & & Lumbago & Infusion & Oral & 7.93 \\
\hline & & & & & Paralysis & & Oral & 25.24 \\
\hline
\end{tabular}


Table 2 Plant species for medicinal purposes in community of Gayasan National Park in Korea (Continued)

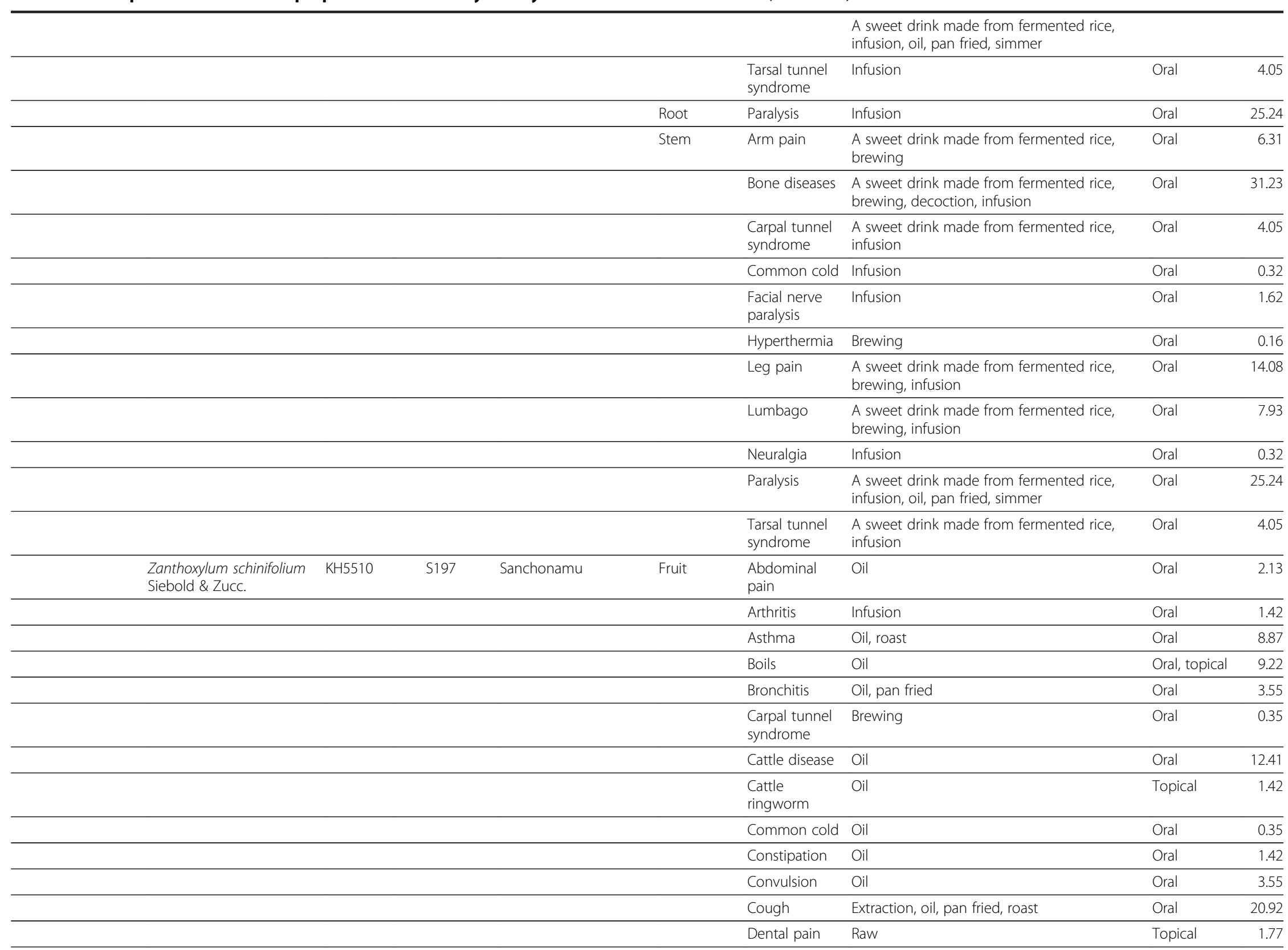


Table 2 Plant species for medicinal purposes in community of Gayasan National Park in Korea (Continued)

\begin{tabular}{|c|c|c|c|c|c|c|c|c|c|}
\hline & & & & & & Diarrhea & Roast & Oral & 1.77 \\
\hline & & & & & & Edema & Oil & Oral, topical & 2.84 \\
\hline & & & & & & Erysipelas & Oil, pan fried & Oral, topical & 4.96 \\
\hline & & & & & & Hoove & Oil & Oral & 1.42 \\
\hline & & & & & & Hypofunction & Oil & Oral & 2.48 \\
\hline & & & & & & Indigestion & Oil & Oral & 0.71 \\
\hline & & & & & & Measles & Oil & Oral & 1.06 \\
\hline & & & & & & Paralysis & Oil & Oral & 3.19 \\
\hline & & & & & & Pig disease & Oil & Oral & 2.84 \\
\hline & & & & & & Pus & Oil & Topical & 1.77 \\
\hline & & & & & & Stomatitis & Oil & Topical & 1.77 \\
\hline & & & & & & $\begin{array}{l}\text { Tarsal tunnel } \\
\text { syndrome }\end{array}$ & Brewing & Oral & 1.06 \\
\hline & & & & & & $\begin{array}{l}\text { Whooping } \\
\text { cough }\end{array}$ & Oil, pan fried & Oral & 1.42 \\
\hline & & & & & Leaf & Snakebite & Rubbing & Topical & 3.90 \\
\hline & & & & & Pine resin & Cattle disease & Dried, powder & Topical & 12.41 \\
\hline & & & & & Stem & Bone diseases & Brewing & Oral & 1.42 \\
\hline & & & & & & Snakebite & Fumigation & Topical & 3.90 \\
\hline \multirow[t]{9}{*}{ Salicaceae } & $\begin{array}{l}\text { Populus maximowiczii A. } \\
\text { Henry }\end{array}$ & KH5511 & S136 & Hwangcheolnamu & Endodermis & $\begin{array}{l}\text { Gastroenteric } \\
\text { disorder }\end{array}$ & Infusion & Oral & 50.00 \\
\hline & & & & & & Leg pain & Infusion & Oral & 50.00 \\
\hline & Salix gracilistyla Miq. & $\mathrm{KH} 5512$ & S162 & Gaetbeodeul & Stem & $\begin{array}{l}\text { Allergic } \\
\text { contact } \\
\text { dermatitis }\end{array}$ & Fumigation & Topical & 33.33 \\
\hline & & & & & & $\begin{array}{l}\text { Lacquer } \\
\text { poisoning }\end{array}$ & Fumigation & Topical & 33.33 \\
\hline & & & & & & Pruritus & Fumigation & Topical & 33.33 \\
\hline & Salix koreensis Andersson & KH5513 & $\mathrm{S} 163$ & Beodeunamu & Bark & Fracture & Raw & Topical & 60.00 \\
\hline & & & & & Fruit & $\begin{array}{l}\text { Lacquer } \\
\text { poisoning }\end{array}$ & Fumigation & Topical & 33.33 \\
\hline & & & & & Stem & $\begin{array}{l}\text { Facial nerve } \\
\text { paralysis }\end{array}$ & Raw & Topical & 6.67 \\
\hline & & & & & & Fracture & Raw & Topical & 60.00 \\
\hline \multirow[t]{3}{*}{ Schisandraceae } & $\begin{array}{l}\text { Schisandra chinensis } \\
\text { (Turcz.) Baill. }\end{array}$ & KH5514 & S169 & Omija & Fruit & Arm pain & Decoction, dissolution, extraction & , Oral & 4.86 \\
\hline & & & & & & Bronchitis & Extraction, infusion & Oral & 5.56 \\
\hline & & & & & & $\begin{array}{l}\text { Poor } \\
\text { circulation }\end{array}$ & Decoction & Oral & 0.69 \\
\hline
\end{tabular}


Table 2 Plant species for medicinal purposes in community of Gayasan National Park in Korea (Continued)

\begin{tabular}{|c|c|c|c|c|c|c|c|c|c|}
\hline & & & & & & Common cold & $\begin{array}{l}\text { A sweet drink made from fermented rice, } \\
\text { boiling, brewing, decoction, dissolution, } \\
\text { dried, extraction, raw, tea }\end{array}$ & Oral & 45.83 \\
\hline & & & & & & Cough & Brewing, decoction, dissolution, extraction & Oral & 15.97 \\
\hline & & & & & & Eye disease & Boiling, dried, extraction & Oral & 6.25 \\
\hline & & & & & & Headache & Boiling, dried & Oral & 3.47 \\
\hline & & & & & & Heart disease & Dried, extraction, tea & Oral & 5.56 \\
\hline & & & & & & $\begin{array}{l}\text { Lack of } \\
\text { energy }\end{array}$ & Extraction & Oral & 2.78 \\
\hline & & & & & & Leg pain & Decoction, dissolution, extraction & Oral & 4.86 \\
\hline & & & & & Stem & $\begin{array}{l}\text { Abdominal } \\
\text { pain }\end{array}$ & Infusion & Oral & 2.08 \\
\hline & & & & & & Anorexia & Infusion & Oral & 2.08 \\
\hline \multirow[t]{16}{*}{ Scrophulariaceae } & Paulownia coreana Uyeki & KH5515 & $S 120$ & Odongnamu & Fruit & Edema & Infusion & Oral & 36.36 \\
\hline & & & & & Stem & Indigestion & Infusion & Oral & 63.64 \\
\hline & $\begin{array}{l}\text { Rehmannia glutinosa } \\
\text { (Gaertn.) Libosch. ex } \\
\text { Steud. }\end{array}$ & KH5516 & S152 & Jihwang & Root & $\begin{array}{l}\text { Abdominal } \\
\text { cold } \\
\text { hypersentivity }\end{array}$ & Pill & Oral & 1.21 \\
\hline & & & & & & Arm pain & Maceration & Topical & 3.64 \\
\hline & & & & & & Bruising & $\begin{array}{l}\text { Brewing, juice, maceration, mixed in liquor, } \\
\text { raw }\end{array}$ & Oral, topical & 29.70 \\
\hline & & & & & & $\begin{array}{l}\text { Carpal tunnel } \\
\text { syndrome }\end{array}$ & Maceration & Topical & 1.21 \\
\hline & & & & & & $\begin{array}{l}\text { Chronic } \\
\text { myofascial } \\
\text { pain }\end{array}$ & Dissolution, maceration & Oral, topical & 6.06 \\
\hline & & & & & & Edema & Maceration, raw & Oral & 1.21 \\
\hline & & & & & & Fracture & Juice, maceration & Oral, topical & 15.15 \\
\hline & & & & & & $\begin{array}{l}\text { Lack of } \\
\text { energy }\end{array}$ & Boiling, decoction & Oral & 3.03 \\
\hline & & & & & & Leg pain & Maceration & Topical & 3.64 \\
\hline & & & & & & Lumbago & Brewing, juice, maceration & Oral, topical & 14.55 \\
\hline & & & & & & Luxation & Maceration & Topical & 4.24 \\
\hline & & & & & & Sprain & Juice, maceration & Oral, topical & 12.73 \\
\hline & & & & & & $\begin{array}{l}\text { Tarsal tunnel } \\
\text { syndrome }\end{array}$ & Maceration & Topical & 1.21 \\
\hline & & & & & & Wound & Maceration & Topical & 2.42 \\
\hline
\end{tabular}


Table 2 Plant species for medicinal purposes in community of Gayasan National Park in Korea (Continued)

\begin{tabular}{|c|c|c|c|c|c|c|c|c|c|}
\hline Selaginellaceae & $\begin{array}{l}\text { Selaginella tamariscina (P. } \\
\text { Beauv.) Spring }\end{array}$ & KH5517 & S172 & Bucheoson & Whole part & Bruising & Maceration & Topical & 26.32 \\
\hline & & & & & & Edema & Maceration & Topical & 26.32 \\
\hline & & & & & & Luxation & Maceration & Topical & 26.32 \\
\hline & & & & & & Sterility & Infusion & Oral & 21.05 \\
\hline \multirow[t]{11}{*}{ Simaroubaceae } & $\begin{array}{l}\text { Picrasma quassioides (D. } \\
\text { Don) Benn. }\end{array}$ & KH5518 & $S 129$ & Sotaenamu & Bark & $\begin{array}{l}\text { Deprived of } \\
\text { mother's milk }\end{array}$ & Maceration & Topical & 32.88 \\
\hline & & & & & & Lumbago & Infusion & Oral & 10.96 \\
\hline & & & & & Leaf & Cattle disease & Infusion & Oral & 19.18 \\
\hline & & & & & Stem & Arthritis & Simmer & Oral & 9.59 \\
\hline & & & & & & Boils & Oil & Topical & 5.48 \\
\hline & & & & & & Cattle disease & Infusion & Oral & 19.18 \\
\hline & & & & & & $\begin{array}{l}\text { Chronic } \\
\text { myofascial } \\
\text { pain }\end{array}$ & Brewing & Oral & 5.48 \\
\hline & & & & & & $\begin{array}{l}\text { Deprived of } \\
\text { mother's milk }\end{array}$ & Infusion, juice, maceration & Topical & 32.88 \\
\hline & & & & & & Hoove & Infusion & Oral & 6.85 \\
\hline & & & & & & Lumbago & Infusion & Oral & 10.96 \\
\hline & & & & & & Pruritus & Infusion & Topical & 9.59 \\
\hline \multirow[t]{5}{*}{ Smilacaceae } & Smilax china L. & KH5519 & S176 & Cheongmiraedeonggul & Rhizome & Hypofunction & Infusion & Topical & 15.00 \\
\hline & & & & & & Leg pain & Infusion & Oral & 30.00 \\
\hline & & & & & & Osteoporosis & Infusion & Oral & 15.00 \\
\hline & & & & & & Pollakiuria & Infusion & Oral & 25.00 \\
\hline & & & & & & Wound & Infusion & Topical & 15.00 \\
\hline \multirow[t]{8}{*}{ Solanaceae } & Capsicum annuum L. & KH5520 & S35 & Gochu & Fruit & $\begin{array}{l}\text { Chronic } \\
\text { myofascial } \\
\text { pain }\end{array}$ & Dissolution & Oral & 100.00 \\
\hline & Lycium chinense Mill. & KH5521 & S105 & Gugijanamu & Fruit & Common cold & Boiling, brewing, decoction, infusion & Oral & 41.46 \\
\hline & & & & & & Eye disease & Boiling & Oral & 12.20 \\
\hline & & & & & & $\begin{array}{l}\text { Gastroenteric } \\
\text { disorder }\end{array}$ & Dried, tea & Oral & 14.63 \\
\hline & & & & & & $\begin{array}{l}\text { Lack of } \\
\text { energy }\end{array}$ & Infusion, tea & Oral & 21.95 \\
\hline & & & & & & Liver diseases & Infusion & Oral & 9.76 \\
\hline & Nicotiana tabacum L. & KH5522 & $\mathrm{S} 110$ & Dambae & Leaf & Snakebite & Infusion & Topical & 100.00 \\
\hline & $\begin{array}{l}\text { Physalis alkekengi var. } \\
\text { francheti (Mast.) Hort }\end{array}$ & KH5523 & S127 & Ggwari & Fruit & Tonsillitis & Infusion & Oral & 100.00 \\
\hline
\end{tabular}


Table 2 Plant species for medicinal purposes in community of Gayasan National Park in Korea (Continued)

\begin{tabular}{|c|c|c|c|c|c|c|c|c|c|}
\hline & Solanum melongena L. & KH5524 & S177 & Gaji & Stem & $\begin{array}{l}\text { Carpal tunnel } \\
\text { syndrome }\end{array}$ & $\begin{array}{l}\text { A sweet drink made from fermented rice, } \\
\text { dried, infusion }\end{array}$ & Oral & 50.00 \\
\hline & & & & & & $\begin{array}{l}\text { Tarsal tunnel } \\
\text { syndrome }\end{array}$ & $\begin{array}{l}\text { A sweet drink made from fermented rice, } \\
\text { dried, infusion }\end{array}$ & Oral & 50.00 \\
\hline & Solanum nigrum L. & KH5525 & S178 & Ggamajung & Aerial part & $\begin{array}{l}\text { Abdominal } \\
\text { pain }\end{array}$ & Infusion & Oral & 4.55 \\
\hline & & & & & & Anorexia & Infusion & Oral & 4.55 \\
\hline & & & & & & Indigestion & Infusion & Oral & 31.82 \\
\hline & & & & & Fruit & Cough & Extraction & Oral & 15.15 \\
\hline & & & & & & Indigestion & Maceration & Oral & 31.82 \\
\hline & & & & & Leaf & Indigestion & Maceration & Oral & 31.82 \\
\hline & & & & & & $\begin{array}{l}\text { Urinary } \\
\text { incontinence }\end{array}$ & Infusion & Oral & 6.06 \\
\hline & & & & & Root & Indigestion & Maceration & Oral & 31.82 \\
\hline & & & & & Stem & Pollakiuria & Infusion & Oral & 6.06 \\
\hline & & & & & & $\begin{array}{l}\text { Urinary } \\
\text { incontinence }\end{array}$ & Infusion & Oral & 6.06 \\
\hline & & & & & Whole part & Cough & Infusion & Oral & 15.15 \\
\hline & & & & & & $\begin{array}{l}\text { Excited } \\
\text { delirium }\end{array}$ & Dried, infusion & Oral & 10.61 \\
\hline & & & & & & Herpes zoster & Infusion & Oral, topical & 21.21 \\
\hline & Solanum tuberosum $\mathrm{L}$. & KH5526 & $S 179$ & Gamja & Tuber & Burn & Maceration, powder, rubbing & Topical & 100.00 \\
\hline \multirow[t]{3}{*}{ Staphyleaceae } & Staphylea bumalda DC. & KH5527 & S183 & Gochunamu & Leaf & $\begin{array}{l}\text { Facial nerve } \\
\text { paralysis }\end{array}$ & Maceration & Topical & 100.00 \\
\hline & & & & & Root & $\begin{array}{l}\text { Facial nerve } \\
\text { paralysis }\end{array}$ & Maceration & Topical & 100.00 \\
\hline & & & & & Stem & $\begin{array}{l}\text { Facial nerve } \\
\text { paralysis }\end{array}$ & Maceration, raw & Topical & 100.00 \\
\hline \multirow[t]{4}{*}{ Sterculiaceae } & $\begin{array}{l}\text { Firmiana simplex (L.) W.F. } \\
\text { Wight }\end{array}$ & KH5528 & $\mathrm{S} 75$ & Byeokodong & Leaf & Leukorrhea & Infusion & Oral & 63.64 \\
\hline & & & & & Stem & Leukorrhea & Infusion & Oral & 63.64 \\
\hline & & & & & & Lumbago & A sweet drink made from fermented rice & Oral & 18.18 \\
\hline & & & & & & Pollakiuria & Infusion & Oral & 18.18 \\
\hline Thelephoraceae & $\begin{array}{l}\text { Sarcodon aspratus (Berk.) } \\
\text { S. Ito }\end{array}$ & KH5529 & S166 & Neungi & Whole part & Afterpain & Infusion & Oral & 100.00 \\
\hline \multirow[t]{3}{*}{ Tricholomataceae } & $\begin{array}{l}\text { Armillariella mellea (Vahl \& } \\
\text { Fr.) Karst. }\end{array}$ & KH5530 & S20 & Bbongnamubeoseot & Whole part & Glycosuria & Infusion & Oral & 100.00 \\
\hline & $\begin{array}{l}\text { Tricholoma matsutake (S. } \\
\text { Ito. et Imai) Sing. }\end{array}$ & KH5531 & S185 & Songi & Whole part & Afterpain & Decoction, infusion & Oral & 57.89 \\
\hline & & & & & & Common cold & Decoction & Oral & 21.05 \\
\hline
\end{tabular}


Table 2 Plant species for medicinal purposes in community of Gayasan National Park in Korea (Continued)

\begin{tabular}{|c|c|c|c|c|c|c|c|c|c|}
\hline & & & & & & Tonsillitis & Infusion & Oral & 21.05 \\
\hline \multirow[t]{28}{*}{ Ulmaceae } & $\begin{array}{l}\text { Ulmus davidiana var. } \\
\text { japonica (Rehder) Nakai }\end{array}$ & KH5532 & S188 & Neureupnamu & Bark & Boils & Dough, dried, maceration, powder & Topical & 15.67 \\
\hline & & & & & & Cancer & Infusion, maceration & Oral, topical & 3.00 \\
\hline & & & & & & Gastric ulcer & $\begin{array}{l}\text { A sweet drink made from fermented rice, } \\
\text { infusion, tea }\end{array}$ & Oral & 2.07 \\
\hline & & & & & & Gastritis & A sweet drink made from fermented rice & Oral & 0.23 \\
\hline & & & & & & $\begin{array}{l}\text { Gastroenteric } \\
\text { disorder }\end{array}$ & Dried, infusion, powder, tea & Oral & 11.29 \\
\hline & & & & & & Hemorrhaging & Powder & Topical & 0.23 \\
\hline & & & & & & Hemorrhoids & Infusion & Topical & 2.76 \\
\hline & & & & & & Indigestion & $\begin{array}{l}\text { A sweet drink made from fermented rice, } \\
\text { infusion }\end{array}$ & Oral & 1.61 \\
\hline & & & & & & Milk fever & Maceration & Oral, topical & 3.92 \\
\hline & & & & & & Pus & Dried, infusion, maceration, powder & Oral, topical & 52.07 \\
\hline & & & & & & Skin diseases & Maceration, powder & Topical & 1.38 \\
\hline & & & & & & Wound & Maceration, powder, raw & Topical & 3.23 \\
\hline & & & & & Endodermis & Boils & Maceration & Topical & 15.67 \\
\hline & & & & & & $\begin{array}{l}\text { Gastroenteric } \\
\text { disorder }\end{array}$ & Infusion & Oral & 11.29 \\
\hline & & & & & & Pus & Maceration & Topical & 52.07 \\
\hline & & & & & Rhizodermis & Boils & Dried, maceration, powder & Topical & 15.67 \\
\hline & & & & & & $\begin{array}{l}\text { Gastroenteric } \\
\text { disorder }\end{array}$ & Infusion & Oral & 11.29 \\
\hline & & & & & & Pus & Maceration & Topical & 52.07 \\
\hline & & & & & & Wound & Maceration, powder & Topical & 3.23 \\
\hline & & & & & Root & Boils & Maceration & Topical & 15.67 \\
\hline & & & & & & Bruising & Maceration & Topical & 0.46 \\
\hline & & & & & & Cancer & Maceration & Topical & 3.00 \\
\hline & & & & & & $\begin{array}{l}\text { Gastroenteric } \\
\text { disorder }\end{array}$ & Dried, infusion & Oral & 11.29 \\
\hline & & & & & & Inflammation & Maceration & Topical & 0.69 \\
\hline & & & & & & Pus & Maceration & Topical & 52.07 \\
\hline & & & & & Stem & Boils & Maceration & Topical & 15.67 \\
\hline & & & & & & Bone diseases & $\begin{array}{l}\text { A sweet drink made from fermented rice, } \\
\text { brewing }\end{array}$ & Oral & 1.38 \\
\hline & & & & & & $\begin{array}{l}\text { Gastroenteric } \\
\text { disorder }\end{array}$ & $\begin{array}{l}\text { A sweet drink made from fermented rice, } \\
\text { boiling, infusion }\end{array}$ & Oral & 11.29 \\
\hline
\end{tabular}


Table 2 Plant species for medicinal purposes in community of Gayasan National Park in Korea (Continued)

\begin{tabular}{|c|c|c|c|c|c|c|c|c|c|}
\hline & & & & & & Milk fever & Maceration & Topical & 3.92 \\
\hline & & & & & & Pus & Dried, maceration, powder & Topical & 52.07 \\
\hline & Ulmus parvifolia Jacq. & KH5533 & S189 & Chamneureupnamu & Bark & Boils & Dough, maceration, powder, raw & Topical & 100.00 \\
\hline Valerianaceae & $\begin{array}{l}\text { Patrinia villosa (Thunb.) } \\
\text { Juss. }\end{array}$ & KH5534 & S119 & Ttukgal & Leaf & Snakebite & Maceration & Topical & 100.00 \\
\hline \multirow[t]{6}{*}{ Violaceae } & $\begin{array}{l}\text { Viola mandshurica W. } \\
\text { Becker }\end{array}$ & KH5535 & S191 & Jebikkot & Aerial part & Bruising & Maceration & Topical & 16.13 \\
\hline & & & & & & Hemorrhage & Rubbing & Topical & 16.13 \\
\hline & & & & & Leaf & Snakebite & Maceration & Topical & 16.13 \\
\hline & & & & & Whole part & $\begin{array}{l}\text { Facial nerve } \\
\text { paralysis }\end{array}$ & Maceration & Topical & 22.58 \\
\hline & & & & & & Sprain & Maceration & Topical & 29.03 \\
\hline & Viola verecunda A.Gray & KH5536 & $\mathrm{S} 192$ & Kongjebikkot & Leaf & Dysentery & Seasoned cooked vegetables & Oral & 100.00 \\
\hline \multirow[t]{7}{*}{ Vitaceae } & $\begin{array}{l}\text { Parthenocissus tricuspidata } \\
\text { (Siebold \& Zucc.) Planch. }\end{array}$ & KH5537 & S118 & Damjaengideonggul & Stem & $\begin{array}{l}\text { Chronic } \\
\text { myofascial } \\
\text { pain }\end{array}$ & Infusion & Oral & 10.00 \\
\hline & & & & & & Glycosuria & Infusion & Oral & 40.00 \\
\hline & & & & & & Lumbago & A sweet drink made from fermented rice & Oral & 40.00 \\
\hline & & & & & & Neuralgia & Decoction & Oral & 10.00 \\
\hline & $\begin{array}{l}\text { Vitis coignetiae Pulliat ex } \\
\text { Planch. }\end{array}$ & KH5538 & S194 & Meoru & Fruit & Asthma & Extraction & Oral & 33.33 \\
\hline & & & & & & Bronchitis & Extraction & Oral & 33.33 \\
\hline & & & & & Stem & Afterpain & Infusion & Oral & 33.33 \\
\hline \multirow[t]{4}{*}{ Zingiberaceae } & Zingiber officinale Roscoe & KH5539 & S199 & Saenggang & Rhizoma & Arm pain & Decoction, dissolution, extraction & Oral & 4.83 \\
\hline & & & & & & Common cold & $\begin{array}{l}\text { Boiling, decoction, dissolution, extraction, } \\
\text { honey, infusion, tea }\end{array}$ & Oral & 67.59 \\
\hline & & & & & & Cough & Decoction, dissolution, extraction, infusion & Oral & 22.76 \\
\hline & & & & & & Leg pain & Decoction, dissolution, extraction & Oral & 4.83 \\
\hline
\end{tabular}


Table 3 Category of ailments and their informant consensus factor (ICF) according to Heinrich et al. [24]

\begin{tabular}{lccc}
\hline Symptom and ailment categories & Taxons & Use citations & ICF \\
\hline Muscular-skeletal disorders & 49 & 2142 & 0.98 \\
\hline Pains & 87 & 2942 & 0.97 \\
\hline Respiratory system disorders & 46 & 1513 & 0.97 \\
\hline Liver complaints & 15 & 447 & 0.97 \\
\hline Cuts and wounds & 20 & 473 & 0.96 \\
\hline Inflammation & 28 & 587 & 0.95 \\
\hline Genitourinary system disorders & 52 & 1062 & 0.95 \\
\hline Circulatory system disorders & 65 & 1250 & 0.95 \\
\hline Skin diseases and disorders & 25 & 464 & 0.95 \\
\hline Gastrointestinal disorders & 68 & 1257 & 0.95 \\
\hline Diabetes & 10 & 165 & 0.95 \\
\hline Nervous system disorders & 34 & 545 & 0.94 \\
\hline Veterinary ailments & 22 & 300 & 0.93 \\
\hline Poisonings & 26 & 321 & 0.92 \\
\hline Others & 36 & 431 & 0.92 \\
\hline Birth-related disorders & 14 & 138 & 0.91 \\
\hline
\end{tabular}

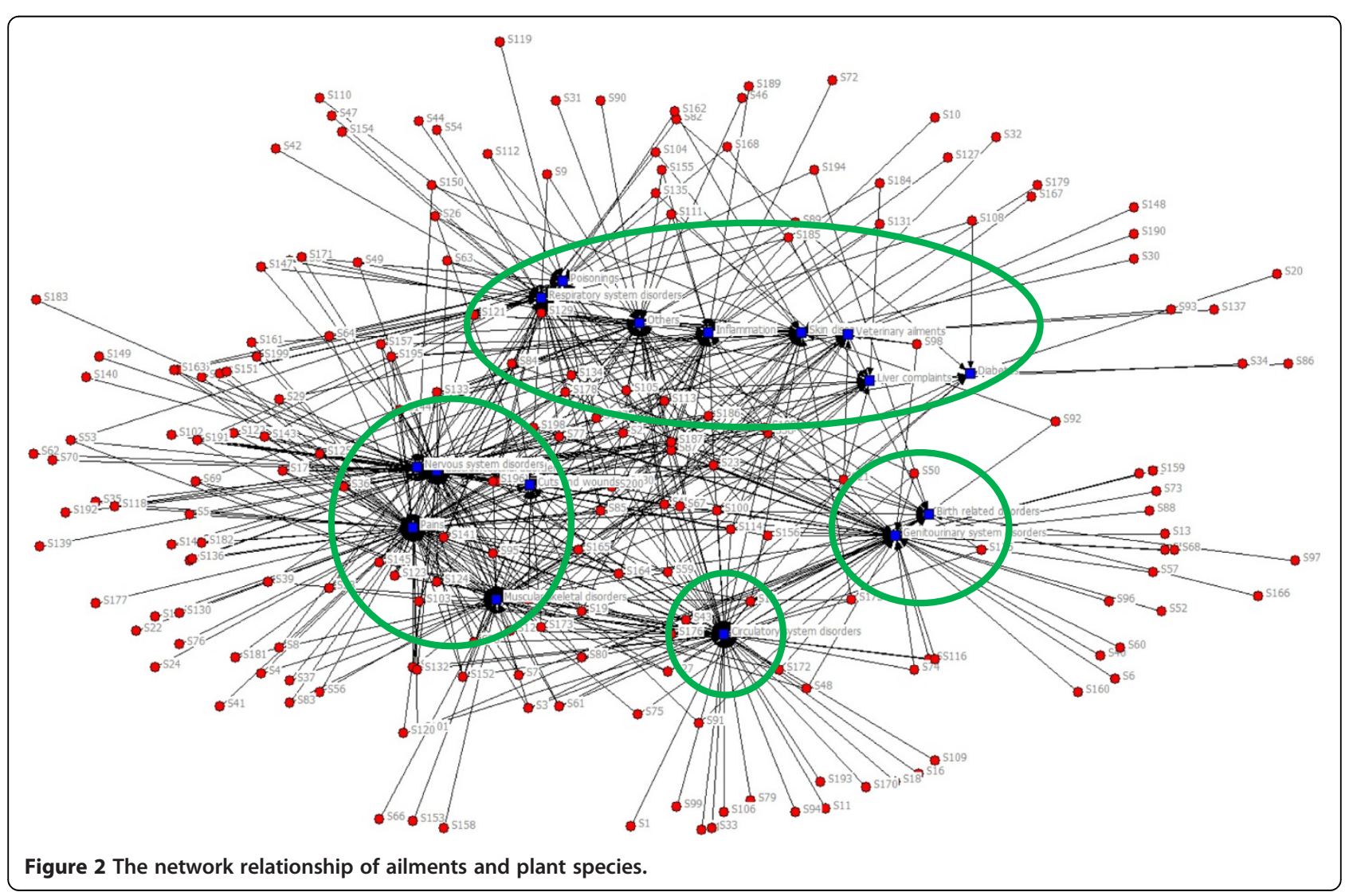

plants used for treating birth related disorders and genitourinary system disorders have a close relationship.

Finally, the circulatory system disorders are the fourth group and are positioned in the bottom section.

\section{Conclusion}

These days, the natural conservation of a national park has focused strongly to preserve bio-resources, which are regarded as vital to its environment. However, the natural conservation of a national park is practically determined by the interrelationship between the bioresources and its utilization by residents.

In relation to this point, this study contains a major focus to obtain the basic data regarding the natural conservation of a plant ecosystem, through the analysis and investigation of traditional knowledge for medicinal plants being used by residents of Gayasan National Park.

Conclusions regarding the preservation of a sustainable natural plant ecosystem within a national park obtained the following results:

First, the high percentage of medicinal plants among flora means that the total amount of medicinal plants gathered by residents may negatively affect the conservation of a natural plant ecosystem within a national park. In order to conserve the plant ecosystem, residents need 
an adaptable health care system for treating their ailments, instead of relying solely on ethnomedicinal therapies.

Second, residents mentioned quite often that they used medicinal plants to treat their own medical conditions. Therefore, it is necessary to prepare a plan of action for conserving the popular medicinal plants.

Third, medicinal plants for treating the category of disease with a higher degree of consensus can be actively harvested for treating medical problems. The over collecting of these medicinal plants will disrupt the plant ecosystem of Gayasan National Park in the near future.

Fourth, 57 species of plants with a FL of 100\% possess a much higher potential for being gathered in the region and it is vital to protect the overuse of these medicinal plants.

Fifth, the results of INA will provide an appropriate plan for the sustainable preservation of a national park through continued study.

Therefore, these particular species need to be conserved for a balanced plant ecosystem within the park.

Consequently, through further study using these results, proper steps need to be established for preparing a wise alternative to create a sustainable natural plant ecosystem for Gayasan National Park and other national parks.

\section{Competing interests}

The authors declare that they have no competing interests.

\section{Authors' contributions}

HK and MJS complied the collected field data, analyzed and draft the manuscript, $\mathrm{HB}, \mathrm{BYL}, \mathrm{CHP}$, and $\mathrm{CWH}$ revised the manuscript added the valuable suggestions for manuscript improvement. All authors read and approved the final manuscript.

\section{Acknowledgements}

This research was supported by a grant from the National Institute of Biological Resources (NIBR), funded by the Ministry of Environment (MOE) of the Republic of Korea (NIBR No. 2012-02-028). The authors are very grateful to all the informants for sharing their orally transmitted traditional knowledge during the fieldwork surveys.

\section{Author details \\ 'Department of Integrated Bioresource Science, Graduate School of Jeonju University, 303 Cheonjam-ro, Wansan-gu, Jeonju 560-759, Republic of Korea. ${ }^{2}$ School of Alternative Medicine and Health Science, Jeonju University, 303 Cheonjam-ro, Wansan-gu, Jeonju 560-759, Republic of Korea. ${ }^{3}$ Department of Biological Resources, National Institute of Biological Resources, Incheon 404-708, Republic of Korea. ${ }^{4}$ School of Liberal Arts, Jeonju University, 303 Cheonjam-ro, Wansan-gu, Jeonju 560-759, Republic of Korea.}

Received: 22 February 2014 Accepted: 14 September 2014 Published: 21 October 2014

\section{References}

1. Parrotta JA, Trosper RL: Traditional Forest Related Knowledge: Sustaining Communities, Ecosystems, and Biocultural Diversity. New York: Springer; 2012

2. Menzies CR: Traditional Ecological Knowledge and Natural Resource Management. Lincoln: University of Mebraska Press; 2006.

3. Unnikrishnan PM, Fadeeva Z: Innovation in Local and Global Learning Systems for Sustainability: Traditional Knowledge and Biodiversity - Learning Contributions of the Regional Centres of Expertise on Education for Sustainable
Development. Yokohama: United Nations University Institute of Advanced Studies; 2013.

4. Unnikrishnan PM, Suneetha MS: Biodiversity, Traditional Knowledge and Community Health: Strengthening Linkages. Singapore: Xpress Pte Ltd.; 2012.

5. Di Sanzo P, De Martino L, Mancini E, De Feo V: Medicinal and useful in the tradition of Rotonda, Pollino National Park, Southern Italy. J Ethnobiol Ethnomed 2013, 9:19. doi:10.1186/1746-4269-9-19.

6. Idolo M, Motti R, Mazzoleni S: Ethnobotanical and phytomedicinal knowledge in a long-history protected area, the Abruzzo, Lazio and Molise National Park (Italian Apennines). J Ethnopharmacol 2010, 127:379-395.

7. Asase A, Hesse DN, Simmonds MS: Uses of multiple plants prescriptions for treatment of malaria by some communities in southern Ghana. J Ethnopharmacol 2012, 144:448-452.

8. Namukobe J, Kasenene JM, Kiremire BT, Byamukama R, KamatenesiMugisha M, Krief S, Dumontet V, Kabasa JD: Traditional plants used for medicinal purposes by local communities around the Northern sector of Kibale

National Park, Uganda. J Ethnopharmacol 2011, 136:236-245.

9. Hoang VS, Pieter B, Paul JAK: Traditional medicinal plants in Ben En National Park, Vietnam. Blumea 2008, 2008(53):569-601.

10. Sharma J, Gairola S, Gaur RD, Painuli RM: The treatment of jaundice with medicinal plants in indigenous communities of the Sub-Himalayan region of Uttarakhanh, India. J Ethnopharmacol 2012, 143:262-291.

11. Estrada E, Villarreal JA, Cantú C, Cabral I, Scott L, Yen C: Ethnobotany in the Cumbrea de Minterrey National Park, Nuevo León, México. J Ethnobiol Ethnomed 2007, 3:8. doi:10.1186/1746-4269-3-8.

12. Thomas E, Semo L, Morales M, Noza Z, Nuñez H, Cayuba A, Noza M, Humaday N, Vaya J, Damme PV: Ethnomedicinal practices and medicinal plant knowledge of the Yuracarés and Trinitarios from Indigenous Territory and National Park Isiboro-Sécure, Bolivian Amazon. J Ethnopharmacol 2011, 133:153-163.

13. Song MJ, Kim H, Heldenbrand B, Jeon JW, Lee SH: Ethnopharmacological survey of medicinal plants in Jeju Island, Korea. J Ethnobiol Ethnomed 2013, 9:48. doi:10.1186/1746-4269-9-48.

14. Korea National Park Service. [http://english.knps.or.kr]

15. You JH, Jeon SK, Seol JW: Flora and conservation plan of Gayasan National Park. J Korean Env Res Tech 2013, 16:109-130.

16. Kim H, Song MJ: Analysis and recordings of orally transmitted knowledge about medicinal plants in the southern mountainous region of Korea. J Ethnopharmacol 2011, 134:676-696.

17. Kim H, Song MJ: Oral traditional knowledge for the treatment of digestive system diseases investigated in North Jeolla Province, Korea. J Med Plant Res 2011, 5:5730-5740.

18. Martin GJ: Ethnobotany: A Conservation Manual. London: Champman \& Hall; 1996.

19. Song M, Kim H: Ethnomedicinal application of plants in the western plain region of North Jeolla Province in Korea. J Ethnopharmacol 2011, 137:167-175.

20. Kim H, Song MJ: Ethnobotany. Seoul: World Science Publisher; 2008.

21. Lee TB: Illustrated Flora of Korea. Seoul: Hyangmunsa; 1979.

22. Lee YN: Flora of Korea. Seoul: Kyohak Publishing Co; 2002.

23. National Knowledge and Information System for Biological Species (NKISBS). [http://www.nature.go.kr:9001/index.do]

24. Heinrich M, Ankli A, Frei B, Weimann C, Sticher O: Medicinal plants in Mexico: healers' consensus and cultural importance. Soc Sci Med 1998, 47:1859-1871.

25. Heinrich M, Edwards S, Moerman DE, Leonti M: Ethnopharmacological field studies: a critical assessment of their conceptual basis and methods. J Ethnopharmacol 2009, 124:1-17.

26. Alexiades MN: Selected Guidelines for Ethnobotanical Research-a Field Manual. Advances in Economic Botany, Volume 10. Bronx: The New York Botanical Garden; 1996.

27. Douglas RW, Johansen UC: Network Analysis and Ethnographic Problems: Process Models of a Turkish Nomad Clan. Idaho: Lexington Books; 2006.

28. Christakis NA, Fowler JH: The spread of obesity in a large social network over 32 years. N Engl J Med 2007, 357:370-379.

29. Christakis NA, Fowler $\mathrm{JH}$ : Social contagion theory: examining dynamic social networks and human behavior. Stat Med 2013, 32:556-577.

30. Christakis NA, Fowler $\mathrm{JH}$ : Rejoinder to commentaries on social contagion theory. Stat Med 2013, 32:597-599. 
31. Borgatti SP: NetDraw Software for Network Visualization. Lexington: Analytic Technologies; 2002.

32. Borgatti SP, Everett MG, Freeman LC: Ucinet for Windows: Software for Social Network Analysis. Harvard: Analytic Technologies; 2002.

33. Srithi K, Balslev H, Wangpakapattanawong P, Srisanga P, Trisonthi C: Medicinal plant knowledge and its erosion among the Mien (Yao) in northern Thailand. J Ethnopharmacol 2009, 123:335-342.

34. Kim YH: Social Inter-Network Analysis. Seoul: Parkyongsa; 2013.

doi:10.1186/1746-4269-10-74

Cite this article as: Song et al: Analysis of traditional knowledge of medicinal plants from residents in Gayasan National Park (Korea). Journal of Ethnobiology and Ethnomedicine 2014 10:74.

\section{Submit your next manuscript to BioMed Central and take full advantage of:}

- Convenient online submission

- Thorough peer review

- No space constraints or color figure charges

- Immediate publication on acceptance

- Inclusion in PubMed, CAS, Scopus and Google Scholar

- Research which is freely available for redistribution 